\title{
REPRESENTATIONS OF AFFINE AND TOROIDAL LIE ALGEBRAS
}

\author{
VYJAYANTHI CHARI
}

\begin{abstract}
We discuss the category $\mathcal{I}$ of level zero integrable representations of loop algebras and their generalizations. The category is not semisimple and so one is interested in its homological properties. We begin by looking at some approaches which are used in the study of other well-known non-semisimple categories in the representation theory of Lie algebras. This is done with a view to seeing if and how far these approaches can be made to work for $\mathcal{I}$. In the later sections we focus first on understanding the irreducible level zero modules and later on certain universal modules, the local and global Weyl modules which in many ways play a role similar to the Verma modules in the BGG-category $\mathcal{O}$. In the last section, we discuss the connections with the representation theory of finite-dimensional associative algebras and on some recent work with J. Greenstein.
\end{abstract}

\section{INTRODUCTION}

In these notes we discuss aspects of the representation theory of affine Kac-Moody Lie algebras. There is a vast literature on this subject and our focus, for the most part, will be on the level zero integrable representations (which include the finite-dimensional representations) of the loop algebra. At the moment, it appears that this direction is the one most likely to generalize to the case of multi-loop algebras and perhaps to extended affine Lie algebras.

A finite-dimensional representation of the loop algebra need not be completely reducible and thus one encounters problems of the type which usually appear in the representation theory of algebraic groups in positive characteristic or in the BGG-category $\mathcal{O}$ for semisimple Lie algebras. However, many of the methods used in the study of the latter subjects are not available for affine Lie algebras. We have tried to illustrate some of the similarities and the difficulties throughout the notes. Thus, we begin with a brief discussion of some of the basic ideas, tools and important results in the representation theory of semisimple Lie algebras and we restrict our attention to ideas that we will use in the later lectures. We then discuss the integrable representations and the BGG-category $\hat{\mathcal{O}}$ for affine algebras, the integer form of the universal enveloping algebra of the affine Lie algebra and the positive level integrable modules. The remaining sections are devoted to integrable level zero modules, the finite-dimensional representations of the loop algebra and generalizations of these results to multiloop algebras. We conclude the notes with a section on the connections between the category of graded representations of a maximal parabolic subalgebra of the affine Lie algebra and representations of associative finite-dimensional algebras, highest weight categories and quivers.

We have tried to maintain some level of informality, reflecting the fact that these are notes of a summer school. This allows for some repetition and topics sometimes appear before they should since they serve as motivation for future lectures. One is also guided by one's own interests and necessarily, these notes do not include many important directions in the study 
of representations of affine Lie algebras: as, for instance, the connections with mathematical physics, number theory, vertex algebras and the relations with the monster group. The interested reader will find, however, that the list of references includes a selection of books and papers dealing with these aspects of the subject.

Acknowledgments. The organizers of the summer school did a superb job of identifying and attracting excellent graduate students and postdoctoral scholars, and I thank them for giving me the opportunity to be a part of the school. Partial support from the NSF grant DMS-0901253 is also acknowledged.

\section{Simple Lie Algebras}

Much of the material in this section can be found in introductory graduate text books [10], [12], [46] on Lie algebras and representation theory. Further references to the literature may be found throughout the section although these are far from comprehensive.

1.1. Let $\mathfrak{g}$ be a finite-dimensional complex simple Lie algebra of rank $n$ with a fixed Cartan subalgebra $\mathfrak{h}$ and let $\Phi$ be the corresponding root system and let $\Pi=\left\{\alpha_{i}: i \in I\right\}$ (where $I=\{1, \cdots, n\}$ ) be a set of simple roots for $\Phi$. The root lattice $Q$ is the $\mathbb{Z}$-span of the simple roots while $Q^{+}$is the $\mathbb{N}$-span of the simple roots, and the set $\Phi^{+}=\Phi \cap Q^{+}$is the set of positive roots in $\Phi$. The restriction of the Killing form $\kappa: \mathfrak{g} \times \mathfrak{g} \rightarrow \mathbb{C}$ to $\mathfrak{h} \times \mathfrak{h}$ induces a nondegenerate bilinear form $($,$) on \mathfrak{h}^{*}$ and we let $\left\{\omega_{1}, \cdots, \omega_{n}\right\} \subset \mathfrak{h}^{*}$ be the fundamental weights given by $2\left(\omega_{j}, \alpha_{i}\right)=\delta_{i, j}\left(\alpha_{i}, \alpha_{i}\right)$. Let $P\left(\operatorname{resp} . P^{+}\right)$be the $\mathbb{Z}$ (resp. $\left.\mathbb{N}\right)$ span of the $\left\{\omega_{i}: 1 \leq i \leq n\right\}$ and note that $Q \subseteq P$. Given $\lambda, \mu \in P$ we say that $\mu \leq \lambda$ iff $\lambda-\mu \in Q^{+}$. Clearly $\leq$ is a partial order on $P$. The set $\Phi^{+}$has a unique maximal element with respect to this order which is denoted by $\theta$ and is called the highest root of $\Phi^{+}$. Set

$$
\rho=\sum_{i=1}^{n} \omega_{i}=\frac{1}{2} \sum_{\alpha \in \Phi^{+}} \alpha .
$$

Let $W$ be the Weyl group of $\mathfrak{g}$ generated by simple refections $\left\{s_{i}: i \in I\right\}$ and let $\ell: W \rightarrow \mathbb{N}$ be the length function which assigns to an element $w \in W$, the length of a reduced expression for $w$ as a product of simple reflections.

1.2. Given $\alpha \in \Phi$, let $\mathfrak{g}_{\alpha}$ be the corresponding root space and set $\mathfrak{n}^{ \pm}=\oplus_{\alpha \in \Phi^{+}} \mathfrak{g}_{ \pm \alpha}$. Then $\mathfrak{n}^{ \pm}$are subalgebras of $\mathfrak{g}$ and we have an isomorphism of vector spaces

$$
\mathfrak{g}=\mathfrak{n}^{-} \oplus \mathfrak{h} \oplus \mathfrak{n}^{+}
$$

For $\alpha \in \Phi^{+}$, fix elements $x_{\alpha}^{ \pm} \in \mathfrak{g}_{ \pm \alpha}$ and $h_{\alpha} \in \mathfrak{h}$ such that they span a Lie subalgebra of $\mathfrak{g}$ which is isomorphic to $\mathfrak{s l}_{2}$, i.e., we have

$$
\left[h_{\alpha}, x_{\alpha}^{ \pm}\right]= \pm 2 x_{\alpha}^{ \pm}, \quad\left[x_{\alpha}^{+}, x_{\alpha}^{-}\right]=h_{\alpha},
$$

and more generally, assume that the set

$$
\left\{x_{\alpha}^{ \pm}:, \alpha \in \Phi^{+}\right\} \cup\left\{h_{\alpha_{i}}: i \in I\right\},
$$

is a Chevalley basis for $\mathfrak{g}$. 
1.3. For any Lie algebra $\mathfrak{a}$, we let $\mathbf{U}(\mathfrak{a})$ be the universal enveloping algebra of $\mathfrak{a}$. Representations of $\mathfrak{a}$ are the same as the representations of $\mathbf{U}(\mathfrak{a})$ and since $\mathbf{U}(\mathfrak{a})$ is a Hopf algebra, one can define the notions of the trivial representation, the tensor product of representations and the dual of a representation. If $\operatorname{dim} \mathfrak{a}<\infty$, then the algebra $\mathbf{U}(\mathfrak{a})$ is Noetherian and this fact plays an important role in the representation theory of the complex semisimple Lie algebras. The Poincare-Birkhoff-Witt theorem implies that we have an isomorphism of vector spaces

$$
\mathbf{U}(\mathfrak{g}) \cong \mathbf{U}\left(\mathfrak{n}^{-}\right) \otimes \mathbf{U}(\mathfrak{h}) \otimes \mathbf{U}\left(\mathfrak{n}^{+}\right) .
$$

Another fact that plays a major role in the representation theory of $\mathfrak{g}$ is that the center $\mathcal{Z}$ of $\mathbf{U}(\mathfrak{g})$ is large and one has a good theory of central characters, as we shall see below. We recall here the definition of the Casimir element $\Omega$ in $\mathcal{Z}$ and we do this in a way that is adapted for use in the affine case. Let $h_{1}^{\prime}, \cdots, h_{n}^{\prime}$ be a basis of $\mathfrak{h}$ which is dual to the basis $h_{\alpha_{1}}, \cdots, h_{\alpha_{n}}$ with respect to the Killing form of $\mathfrak{g}$ and set

$$
\Omega=\sum_{i=1}^{n} h_{i} h_{i}^{\prime}+h_{\rho}+2 \sum_{\alpha \in \Phi^{+}} x_{\alpha}^{-} x_{\alpha}^{+},
$$

where $h_{\rho} \in \mathfrak{h}$ is defined by requiring $\rho(h)=\kappa\left(h, h_{\rho}\right)$.

1.4. Recall that a $\mathfrak{g}$-module $V$ is said to be a weight module if

$$
V=\bigoplus_{\mu \in \mathfrak{h}^{*}} V_{\mu}
$$

where $V_{\mu}=\{v \in V: h v=\mu(h) v \quad h \in \mathfrak{h}\}$. An element $\mu \in \mathfrak{h}^{*}$ is a weight of $V$ if $V_{\mu} \neq 0$ and we set $\operatorname{wt}(V)=\left\{\mu \in \mathfrak{h}^{*}: V_{\mu} \neq 0\right\}$.

The BGG-category $\mathcal{O}$ is the full subcategory of $\mathfrak{g}$-modules $M$ satisfying the condition that $M$ is a finitely generated weight module which is $\mathfrak{n}^{+}$-locally finite (for all $m \in M$ we have $\left.\operatorname{dim} \mathbf{U}\left(\mathfrak{n}^{+}\right) m<\infty\right)$ as a $\mathfrak{n}^{+}$-module. The morphisms in $\mathcal{O}$ are just the $\mathfrak{g}$-module maps. The following is straightforward.

Lemma. The category $\mathcal{O}$ is abelian and all objects of $\mathcal{O}$ are Noetherian modules for $\mathbf{U}(\mathfrak{g})$. Moreover if $M \in \mathcal{O}$ then

$$
\operatorname{dim} M_{\mu}<\infty \text { for all } \mu \in \mathfrak{h}^{*},
$$

and there exist finitely many elements $\mu_{1}, \cdots, \mu_{k} \in \mathfrak{h}^{*}$ (depending on $M$ ) such that

$$
\text { wt } M \subset \bigcup_{s=1}^{k}\left(\mu_{s}-Q^{+}\right) \text {. }
$$

The formal character $\operatorname{ch}(V)$ of a weight module $V$, encodes the information on the dimensions of the weight spaces, i.e., it is the function $\mathfrak{h}^{*} \rightarrow \mathbb{N}$ which sends $\lambda$ to $\operatorname{dim} V_{\lambda}$. This is usually written as follows. Given $\lambda \in \mathfrak{h}^{*}$ let $e(\lambda): \mathfrak{h}^{*} \rightarrow \mathbb{Z}$ be defined by $e(\lambda) \lambda=1, e(\lambda) \mu=0$ if $\mu \neq \lambda$, then

$$
\operatorname{ch}(V)=\sum_{\mu \in \mathfrak{h}^{*}} \operatorname{dim} V_{\mu} e(\mu) .
$$

Formal characters behave well with respect to direct sums and tensor products. 
1.5. An important family of objects in $\mathcal{O}$ are the highest weight modules. A module $M$ is said to be of highest weight $\lambda$ with highest weight vector $m$ if $M=\mathbf{U}(\mathfrak{g}) m$ and

$$
h m=\lambda(h) m, \quad x_{\alpha}^{+} m=0, \quad h \in \mathfrak{h}, \quad \alpha \in \Phi^{+} .
$$

For $\lambda \in \mathfrak{h}^{*}$ let $M(\lambda)=\mathbf{U}(\mathfrak{g}) m_{\lambda}$ be the universal highest weight module (also called the Verma module) with highest weight $\lambda$ and highest weight vector $m_{\lambda}$, in other words the equation (1.5) gives the defining relations of $m_{\lambda}$. Using (1.2), it is straightforward to show that

$$
\text { wt } M(\lambda)=\lambda-Q^{+}, \quad \operatorname{dim} M(\lambda)_{\lambda}=1, \quad \operatorname{dim} M(\lambda)_{\mu}<\infty, \quad \mu \in \mathfrak{h}^{*} .
$$

In particular, it follows that $M(\lambda)$ has a unique irreducible quotient $V(\lambda)$ and using Lemma 1.4, we get

Lemma. Any irreducible object in $\mathcal{O}$ is isomorphic to $V(\lambda)$ for some $\lambda \in \mathfrak{h}^{*}$.

1.6. The most obvious family of $\mathfrak{g}$-modules which are in $\mathcal{O}$ are of course the finitedimensional ones and the following result completely describes the corresponding full subcategory of $\mathcal{O}$.

\section{Theorem.}

(i) Any finite-dimensional $\mathfrak{g}$-module is isomorphic to a direct sum of irreducible modules, i.e., the full subcategory of $\mathcal{O}$ consisting of finite-dimensional modules is semisimple.

(ii) For $\lambda \in \mathfrak{h}^{*}$, the irreducible module $V(\lambda)$ is finite-dimensional iff $\lambda \in P^{+}$and the character of $V(\lambda)$ is given by the Weyl-character formula,

$$
\left(\sum_{w \in W}(-1)^{\ell(w)} e(w \rho)\right) \operatorname{ch}(V(\lambda))=\sum_{w \in W}(-1)^{\ell(w)} e(w(\lambda+\rho)) .
$$

(iii) For $\lambda \in P^{+}$, the module $V(\lambda)$ is generated by an element $v_{\lambda}$ with defining relations:

$$
h v_{\lambda}=\lambda(h) v_{\lambda}, \quad x_{\alpha_{i}}^{+} v_{\lambda}=0, \quad\left(x_{\alpha_{i}}^{-}\right)^{\lambda\left(h_{\alpha_{i}}\right)+1} v_{\lambda}=0,
$$

where $h \in \mathfrak{h}$ and $1 \leq i \leq n$.

We shall say that a $\mathfrak{g}$-module $V$ is locally finite-dimensional if it isomorphic to a sum of finite-dimensional modules. The preceding theorem implies that $V$ is isomorphic to a direct sum of modules $V(\lambda), \lambda \in P^{+}$. If in addition, we have

$$
\operatorname{dim} \operatorname{Hom}_{\mathfrak{g}}(V(\lambda), V)<\infty \quad \lambda \in P^{+},
$$

then we can define a function $P^{+} \rightarrow \mathbb{N}$ which maps $\lambda$ to $\operatorname{dim} \operatorname{Hom}_{\mathfrak{g}}(V(\lambda), V)$. We denote this function by $\operatorname{ch}_{\mathfrak{g}}(V)$ and observe that it encodes the multiplicity of an irreducible representation in $V$ (whereas $\operatorname{ch}(V)$ encodes the dimension of a weight space). Clearly, we can write,

$$
\operatorname{ch}_{\mathfrak{g}}(V)=\sum_{\lambda \in P^{+}} \operatorname{dim} \operatorname{Hom}_{\mathfrak{g}}(V(\lambda), V) e(\lambda),
$$

where $e(\lambda)$ is the function (or rather its restriction to $P^{+}$) defined in Section 1.4. 
1.7. The category $\mathcal{O}$ itself is not semisimple, for instance the Verma-modules are indecomposable but not always irreducible and we give an example of this, in the case of $\mathfrak{s l}_{2}$. Let $x, y, h$ be the standard basis of $\mathfrak{s l}_{2}$. Since $\mathfrak{h}$ is one-dimensional, we identify the set $P^{+}$with $\mathbb{N}$ and consider the Verma-module $M(0)$ and its highest weight vector $m_{0}$. It is straightforward to see that the element $y m_{0} \in M(0)$ generates a highest weight submodule isomorphic to $M(-2)$ and one has the following non-split short exact sequence of $\mathfrak{s l}_{2}$-modules:

$$
0 \rightarrow M(-2) \rightarrow M(0) \rightarrow \mathbb{C} \rightarrow 0 .
$$

This lack of semi-simplicity has resulted in the development of many approaches designed to understand the structure of $\mathcal{O}$ and we briefly sketch a few directions. The interested reader could refer to [47] and to the references in that book. In the later sections of these notes, we shall see that some of these approaches can or may be adapted to work for affine Lie algebras while others will clearly have no parallel for infinite-dimensional Lie algebras.

1.8. We start with the theory of central characters as an example of something that is very powerful in the study of $\mathcal{O}$ but is essentially not available for affine Lie algebras. A central character is a homomorphism of algebras $\chi: \mathcal{Z} \rightarrow \mathbb{C}$ (where $\mathcal{Z}$ is the center of $\mathbf{U}(\mathfrak{g})$ ) and a $\mathfrak{g}$-module $M$ is said to admit a central character $\chi$ if:

$$
z m=\chi(z) m, \quad z \in \mathcal{Z}, \quad m \in M .
$$

If $z \in \mathcal{Z}$, one can prove without too much difficulty that there exists a unique element $\beta(z) \in \mathbf{U}(\mathfrak{h})$ such that $z-\beta(z) \in \mathfrak{n}^{-} \mathbf{U}(\mathfrak{g}) \mathfrak{n}^{+}$. The corresponding map $\beta: \mathcal{Z} \rightarrow \mathbf{U}(\mathfrak{h})$ is an algebra homomorphism and is called the Harish-Chandra homomorphism. Suppose now that $\lambda \in \mathfrak{h}^{*}$ and let $m \in M(\lambda)$, say $m=g m_{\lambda}$ for some $g \in \mathbf{U}(\mathfrak{g})$. One has

$$
z m=g z m_{\lambda}=\lambda(\beta(z)) g m_{\lambda},
$$

where $\lambda \in \mathfrak{h}^{*}$ is extended to an algebra homomorphism also denoted $\lambda: \mathbf{U}(\mathfrak{h}) \rightarrow \mathbb{C}$. It is clear that the composite map $\chi_{\lambda}: \mathcal{Z} \rightarrow \mathbb{C}$ sending $z \rightarrow \lambda(\beta(z))$ is an algebra homomorphism and hence $M(\lambda)$ admits a central character.

It is now natural to ask if the $\chi_{\lambda}$ are all distinct and if any homomorphism from $\mathcal{Z} \rightarrow \mathbb{C}$ is of the form $\chi_{\lambda}$ for some $\lambda \in \mathfrak{h}^{*}$. The answer to the first question is quite clearly no. Consider the example given in (1.8). Since $M(0)$ admits a central character any submodule or quotient of $M(0)$ also has the same central character and hence we get in this case that $\chi_{0}=\chi_{-2}$. It is a theorem of Harish-Chandra, that for $\lambda, \mu \in \mathfrak{h}^{*}$ :

$$
\chi_{\lambda}=\chi_{\mu} \Longleftrightarrow \lambda+\rho=w(\mu+\rho) \text { for some } w \in W,
$$

and also that the answer to the second question above is yes. As a consequence of this theorem one can show that:

Theorem. Any module $M \in \mathrm{ObO}$ is Artinian. In particular, $M$ has a Jordan-Hölder series and can be written uniquely (up to isomorphism and re-indexing) as a direct sum of indecomposable modules.

For $M \in \mathcal{O}$ and $\mu \in \mathfrak{h}^{*}$ denote by $[M: V(\mu)]$ the multiplicity of $V(\mu)$ in the Jordan-Hölder series of $M$. The problem of determining the multiplicities of the composition factors of the Verma module is a very difficult one and the answer is given by the famous Kazhdan-Lusztig conjecture [55] in terms of the Kazhdan-Lusztig (KL) polynomials. The conjecture is now 
a theorem due to Beilinson-Bernstein [4] and independently Brylinski-Kashiwara [8]. The KL-polynomials are defined recursively and explicit computations using the definition are formidable. There is extensive literature devoted to understanding the combinatorics of the Kazhdan-Lusztig polynomials and to developing programs to compute the polynomials.

1.9. It is natural to consider the homological properties of $\mathcal{O}$ by asking questions such as: does $\mathcal{O}$ contain enough projectives? In fact it does and one can determine all indecomposable projective objects in $\mathcal{O}$. These are again indexed by elements of $\mathfrak{h}^{*}$ and we denote by $P(\lambda)$ the corresponding indecomposable projective module. The module $P(\lambda)$ has a Verma flag which is a filtration in which the subsequent quotients are Verma-modules. As in the case of Jordan-Hölder series of a module, the length of any two filtrations of $P(\lambda)$ by Verma modules is the same and so is the multiplicity of a Verma module $M(\mu)$ in any two filtrations.

And this allows us now to state the fundamental result proved by Bernstein-Gelfand and Gelfand:

Theorem. For $\lambda, \mu \in \mathfrak{h}^{*}$ we have

$$
[P(\lambda): M(\mu)]=[M(\mu): V(\lambda)],
$$

where $[P(\lambda): M(\mu)]$ is the multiplicity of $M(\mu)$ in a Verma flag of $P(\lambda)$ and $[M(\mu): V(\lambda)]$ is the multiplicity of $V(\mu)$ in the Jordan-Hölder series of $M(\mu)$.

Much of the work on $\mathcal{O}$ including the Kazhdan-Lusztig conjecture was stimulated by this result.

1.10. One could try to understand $\mathcal{O}$ by looking at smaller, more manageable subcategories of $\mathcal{O}$ and this is done as follows. It is not true that the center always acts on objects (even indecomposable ones) of $\mathcal{O}$ via a central character. However, since $\mathcal{Z}$ is commutative and we are working over the complex numbers it is true that any $M \in \mathrm{ObO}$ can be written as a direct sum of generalized eigenspaces for the action of $\mathcal{Z}$. Hence if $M$ is indecomposable then there exists an algebra homomorphism $\chi: \mathcal{Z} \rightarrow \mathbb{C}$ such that

$$
M=M^{\chi}=\left\{m \in M:(z-\chi(z))^{k} m=0 \text { for some } k=k(m) \in \mathbb{N}\right\} .
$$

Moreover in this case, if $V(\mu)$ occurs in a Jordan-Hölder series for $M$ then $\chi=\chi_{\mu}$. This motivates the following definition. Let $\mathcal{O}_{\chi}$ be the full subcategory of $M$ consisting of $M \in$ $\mathrm{Ob} \mathcal{O}$ such that $M=M^{\chi}$. Using (1.9) we see that $\mathcal{O}_{\chi}$ has only finitely many simple objects $V(\mu)$ with $\chi_{\mu}=\chi$ and it suffices to understand $\mathcal{O}_{\chi}$. The endomorphism algebra of the projective generator of $\mathcal{O}_{\chi}$ is a finite-dimensional associative algebra $\mathcal{A}_{\chi}$ and its (left)-module category is equivalent to $\mathcal{O}_{\chi}$. The category $\mathcal{O}_{\chi}$ fits into the axiomatic framework of highest weight categories developed by Cline, Parshall and Scott, 34 and $\mathcal{A}_{\chi}$ is quasi-hereditary. The algebra $\mathcal{A}_{\chi}$ is given by a quiver with relations although it is in general very hard to compute these relations, see however [71] where an algorithm to compute these relations is given. Also, under suitable conditions [3], [70], the algebra $\mathcal{A}_{\chi}$ has a Koszul grading and hence the homological properties of $\mathcal{O}_{\chi}$ can also be understood from this viewpoint.

1.11. Before we leave the realm of finite-dimensional simple Lie algebras, we discuss the Kostant $\mathbb{Z}$-form of $\mathbf{U}(\mathfrak{g})$ and the passage to positive characteristic. Given any associative algebra $A$ over $\mathbb{C}$ and elements $a \in A$ and $r \in \mathbb{N}$, the $r^{t h}$-divided power of $a$ is the element $a^{(r)}=a^{r} / r$ !. Let $\mathbf{U}_{\mathbb{Z}}(\mathfrak{g})$ be the $\mathbb{Z}$-subalgebra of $\mathbf{U}(\mathfrak{g})$ generated by the elements $\left(x_{\alpha}^{ \pm}\right)^{(r)}$, 
$\alpha \in \Phi^{+}, r \in \mathbb{N}$ and define $\mathbf{U}_{\mathbb{Z}}\left(\mathfrak{n}^{ \pm}\right)$in the obvious way. To define the analog of the Cartan subalgebra, one does not do the obvious and take the divided powers of the $h_{i}, 1 \leq i \leq n$. Instead, one is guided by the following formula which rewrites the product $\left(x_{\alpha}^{+}\right)^{(s)}\left(x_{\alpha}^{-}\right)^{(r)}$ in Poincare-Birkhoff-Witt order as in (1.2),

$$
\left(x_{\alpha}^{+}\right)^{(s)}\left(x_{\alpha}^{-}\right)^{(r)}=\sum_{m=0}^{\min (r, s)}\left(x_{\alpha}^{-}\right)^{(r-m)}\left(\begin{array}{c}
h_{\alpha}-r-s+2 m \\
m
\end{array}\right)\left(x_{\alpha}^{+}\right)^{(s-m)},
$$

where for any $m \in \mathbb{N}$ and $h \in \mathfrak{h}$, we set

$$
\left(\begin{array}{l}
h \\
m
\end{array}\right)=\frac{h(h-1) \cdots(h-m+1)}{m !} .
$$

We then define $\mathbf{U}_{\mathbb{Z}}(\mathfrak{h})$ to be the $\mathbb{Z}$-subalgebra generated by the elements $\left(\begin{array}{c}h_{\alpha_{i}} \\ m\end{array}\right), 1 \leq i \leq n$, $m \in \mathbb{N}$. It is a theorem of Kostant that $\mathbf{U}_{\mathbb{Z}}(\mathfrak{g})$ has a PBW-basis, by which we mean a $\mathbb{Z}$-basis of $\mathbf{U}_{\mathbb{Z}}(\mathfrak{h})$ consisting of all ordered monomials from the set

$$
\left\{\left(x_{\alpha}^{ \pm}\right)^{(r)},\left(\begin{array}{c}
h_{i} \\
r
\end{array}\right): \alpha \in \Phi^{+}, \quad i \in I, r \in \mathbb{N}\right\} .
$$

This means that $\mathbf{U}_{\mathbb{Z}}(\mathfrak{g})$ is a $\mathbb{Z}$-lattice in $\mathbf{U}(\mathfrak{g})$ and moreover one can prove that if $\lambda \in P^{+}$, and we set

$$
V_{\mathbb{Z}}(\lambda)=\mathbf{U}_{\mathbb{Z}}(\mathfrak{g}) v_{\lambda}
$$

then $V_{\mathbb{Z}}(\lambda)$ is also a $\mathbb{Z}$-lattice in $V(\lambda)$. Let $\mathbb{F}$ be a field of characteristic $p$ and regard $\mathbb{F}$ as a left module for $\mathbb{Z}$. Set

$$
\mathbf{U}_{\mathbb{F}}(\mathfrak{g})=\mathbf{U}_{\mathbb{Z}}(\mathfrak{g}) \otimes_{\mathbb{Z}} \mathbb{F}, \quad V_{\mathbb{F}}(\lambda)=V_{\mathbb{Z}}(\lambda) \otimes_{\mathbb{Z}} \mathbb{F} .
$$

Clearly, $\mathbf{U}_{\mathbb{F}}(\mathfrak{g})$ is an associative algebra over $\mathbb{F}$ (called the hyperalgebra of $\mathfrak{g}$ over $\mathbb{F}$ ) and $V_{\mathbb{F}}(\lambda)$ is an indecomposable (usually reducible) module for this algebra, and is called the Weyl module of weight $\lambda$. There is extensive literature on the study of finite-dimensional representations of the hyperalgebra. One can talk about weight modules (whose definition requires a natural modification) and characters of these modules and we summarize the relevant points for our next lectures in the following theorem.

Theorem. (i) Given $\lambda \in P^{+}$, the module $V_{\mathbb{F}}(\lambda)$ has a unique irreducible quotient and any irreducible finite-dimensional module of $\mathbf{U}_{\mathbb{F}}(\mathfrak{g})$ is isomorphic to such a quotient for some $\lambda \in P^{+}$.

(ii) The character of $V_{\mathbb{F}}(\lambda)$ is given by the Weyl character formula.

(iii) The module $V_{\mathbb{F}}(\lambda)$ is generated by the element $v_{\lambda} \otimes 1$ and the defining relations are:

$$
\left(x_{\alpha}^{+}\right)^{(r)}\left(v_{\lambda} \otimes 1\right)=0, \quad\left(\begin{array}{c}
h_{i} \\
r
\end{array}\right)\left(v_{\lambda} \otimes 1\right)=\left(\begin{array}{c}
\lambda\left(h_{i}\right) \\
r
\end{array}\right)\left(v_{\lambda} \otimes 1\right), \quad\left(x_{\alpha}^{-}\right)^{(s)}\left(v_{\lambda} \otimes 1\right)=0,
$$

for all $\alpha \in \Phi^{+}, i \in I, r, s \in \mathbb{N}$ with $s \geq \lambda\left(h_{i}\right)+1$.

Note that the fact that the modules $V_{\mathbb{F}}(\lambda)$ are indecomposable but not irreducible implies that the category of finite-dimensional representations of the hyperalgebra is also not semisimple and many of the problems and methods discussed for the category $\mathcal{O}$ are also studied and used in this case. 


\section{Affine Lie algebras}

We now turn our attention to the case of the best understood Kac-Moody Lie algebras: the affine Lie algebras. There are two definitions available for these algebras: one is via a finite set of generators and relations and the other is very explicit as we shall see below. It is the interplay between these two definitions that makes the study of these algebras and their representations tractable. The explicit realization is what we need for our purposes and so we refer the reader to [12], [53] for the presentation via Chevalley generators and relations. Most of the results discussed in this section can also be found in those two books. We use freely the notation of the first section.

2.1. Let $t$ be an indeterminate and $\mathbb{C}\left[t, t^{-1}\right]$ the algebra of Laurent polynomials in $t$ with the coefficients in $\mathbb{C}$. For any complex Lie algebra $\mathfrak{a}$, the loop algebra $L(\mathfrak{a})$ is defined by,

$$
L(\mathfrak{a})=\mathfrak{a} \otimes \mathbb{C}\left[t, t^{-1}\right]
$$

with Lie bracket,

$$
[a \otimes f(t), b \otimes g(t)]=[a, b] \otimes f(t) g(t), \quad a, b \in \mathfrak{a}, \quad f, g \in \mathbb{C}\left[t, t^{-1}\right] .
$$

The algebra $\mathfrak{a}$ is identified with the subalgebra $\mathfrak{a} \otimes 1$ and we shall assume this from now on without further mention. Given an automorphism $\sigma$ of $\mathfrak{a}$ of order $m$ and $\zeta$ a fixed primitive $m^{\text {th }}$ root of unity, we can write,

$$
\mathfrak{a}=\bigoplus_{s=0}^{m-1} \mathfrak{a}_{s}, \quad \mathfrak{a}_{s}=\left\{a \in \mathfrak{a}: \sigma(a)=\zeta^{s} a\right\},
$$

and we set

$$
L(\mathfrak{a}, \sigma, m)=\bigoplus_{s=0}^{m-1} \mathfrak{a}_{s} \otimes t^{s} \mathbb{C}\left[t^{m}, t^{-m}\right] .
$$

It is a simple matter to check that $L(\mathfrak{a}, \sigma, m)$ is a Lie subalgebra of $L(\mathfrak{a})$ and it is a proper subalgebra if and only if $\sigma$ is not the identity automorphism of $\mathfrak{a}$, and in this case, we call $L(\mathfrak{a}, \sigma, m)$ a twisted loop algebra.

2.2. The Lie algebra $L(\mathfrak{g})$ admits a unique (up to an isomorphism) non-trivial central extension which we denote by $\tilde{L}(\mathfrak{g})$. It can be constructed as follows: set

$$
\tilde{L}(\mathfrak{g})=L(\mathfrak{g}) \oplus \mathbb{C} c
$$

and define the Lie bracket by requiring $c$ to be central and by setting

$$
\left[x \otimes t^{p}, y \otimes t^{r}\right]=[x, y] \otimes t^{p+r}+p \delta_{p+r, 0} \kappa(x, y) c, \quad x, y \in \mathfrak{g}, \quad p, r \in \mathbb{Z},
$$

where $\delta_{p+r, 0}$ is the Kronecker $\delta$ symbol and recall that $\kappa$ is the Killing form of $\mathfrak{g}$. The derivation $d=t d / d t$ of $\mathbb{C}\left[t, t^{-1}\right]$ acts on $L(\mathfrak{g})$ and on $\tilde{L}(\mathfrak{g})$ by

$$
d\left(x \otimes t^{r}\right)=r x \otimes t^{r}, \quad[d, c]=0 .
$$

The semi-direct product Lie algebra

$$
\hat{L}(\mathfrak{g})=\tilde{L}(\mathfrak{g}) \oplus \mathbb{C} d,
$$

is called the untwisted affine Lie algebra and $\mathfrak{g}$ is naturally isomorphic to a subalgebra of $\hat{L}(\mathfrak{g})$. If $\sigma$ is a Dynkin diagram automorphism of $\mathfrak{g}$, then $\tilde{L}(\mathfrak{g}, \sigma, m)$ and $\hat{L}(\mathfrak{g}, \sigma, m)$ are defined in the 
obvious way and are Lie subalgebras of $\tilde{L}(\mathfrak{g})$ and $\hat{L}(\mathfrak{g})$ respectively. The algebra $\hat{L}(\mathfrak{g}, \sigma, m)$ is called a twisted affine Lie algebra.

For the most part, we will deal only with the untwisted affine Lie algebras and we will refer to these simply as affine Lie algebras. We will usually indicate if the results we discuss are known for for the twisted affine algebras.

2.3. The affine Lie algebra comes equipped with a naturally defined root system and a set of simple roots. Set $\hat{\mathfrak{h}}=\mathfrak{h} \oplus \mathbb{C} c \oplus \mathbb{C} d$ and define $\delta \in \hat{\mathfrak{h}}^{*}$ by $\delta(d)=1, \delta(\mathfrak{h} \oplus \mathbb{C} c)=0$. Then $\hat{\mathfrak{h}}$ is abelian and given $\mu \in \mathfrak{h}^{*}$ we extend $\mu$ to an element of $\hat{\mathfrak{h}}^{*}$ by requiring

$$
\mu(d)=0, \quad \mu(c)=0,
$$

and by abuse of notation, we continue to denote this element by $\mu$. The adjoint action of $\hat{\mathfrak{h}}$ on $\hat{L}(\mathfrak{g})$ is semisimple and the non-zero eigenvalues (the set of roots) are,

$$
\hat{\Phi}=\{\alpha+r \delta: \alpha \in \Phi, r \in \mathbb{Z}\} \bigsqcup\{r \delta: r \in \mathbb{Z}, \quad r \neq 0\} .
$$

The corresponding eigenspaces (root spaces) are $\mathfrak{g}_{\alpha} \otimes t^{s}, \mathfrak{h} \otimes t^{r}$ where $\alpha \in \Phi, r, s \in \mathbb{Z}$ and $r \neq 0$. Notice that the eigenspaces corresponding to $r \delta$ are of dimension $\operatorname{dim} \mathfrak{h}$ and hence bigger than one in general. Setting

$$
\hat{\Pi}=\left\{\alpha_{i}: 0 \leq i \leq n\right\}, \quad \alpha_{0}=-\theta+\delta,
$$

we find that any element of $\hat{\Phi}$ can be written uniquely as an integer linear combination of elements of $\hat{\Pi}$ where the coefficients are all either non-negative or non-positive. Elements of the set $\hat{\Pi}$ are called simple roots and we have

$$
\hat{\Phi}^{+}=\left\{ \pm \alpha+r \delta: r \in \mathbb{N}_{+}, \quad \alpha \in \Phi\right\} \quad \sqcup \Phi^{+} \quad \sqcup\left\{r \delta: r \in \mathbb{N}_{+}\right\} .
$$

The subalgebras $\hat{\mathfrak{n}}^{ \pm}$are defined in the natural way and one has triangular decompositions similar to the ones given in (1.1) and (1.2) for $\mathfrak{g}$. Set

$$
x_{\theta}^{\mp} \otimes t^{ \pm 1}=x_{\alpha_{0}}^{ \pm}, \quad h_{0}=c-h_{\theta} .
$$

The elements $x_{\alpha_{i}}^{ \pm}, h_{\alpha_{i}} 0 \leq i \leq n$ are called the Chevalley generators of $\hat{L}(\mathfrak{g})$ and an abstract presentation of the Lie algebra in terms of these generators can be given. The fundamental weights $\Lambda_{i}, 0 \leq i \leq n$ are elements of $\hat{\mathfrak{h}}^{*}$ defined by $\Lambda_{i}\left(h_{\alpha_{j}}\right)=\delta_{i, j}$. The notion of root lattice $\hat{Q}$, weight lattice $\hat{P}$, weight modules, weight spaces, highest weight modules and so on, have obvious analogs in the affine case, one just replaces $\mathfrak{h}$ by $\hat{\mathfrak{h}}$ and so on. The Killing form of $\hat{L}(\mathfrak{g})$ cannot be defined in the usual way since $\hat{L}(\mathfrak{g})$ is infinite-dimensional. However, the formulae

$$
\begin{gathered}
<x \otimes t^{r}, y \otimes t^{s}>=\kappa(x, y) \delta_{r+s, 0}, \quad<x \otimes t^{r}, \mathbb{C} c \oplus \mathbb{C} d>=0 \\
<c, c>=<d, d>=0, \quad<c, d>=1
\end{gathered}
$$

define an invariant symmetric nondegenerate bilinear form on $\hat{L}(\mathfrak{g})$. The affine Weyl group $\hat{W}$ is the subgroup of $\operatorname{Aut}\left(\mathfrak{h}^{*} \oplus \mathbb{C} \delta\right)$ generated by the simple reflections $\left\{s_{i}: i=0, \cdots, n\right\}$ and is an infinite Coxeter group. As for finite-dimensional simple Lie algebras, the length of an element $w$ in $\hat{W}$ is just the number of positive roots which are turned negative by $w$ : in particular (unlike in the situation for finite-dimensional simple Lie algebras) there does not exist an element in $\hat{W}$ which maps $\hat{\Phi}^{+}$to $\hat{\Phi}^{-}$. 
The many parallels with the structure of $\mathfrak{g}$ suggest that the study of the representation theory of $\hat{L}(\mathfrak{g})$ should proceed in the same way as the study of representations of $\mathfrak{g}$. However, the differences noted above, together with the observation that $\mathbf{U}(\hat{L}(\mathfrak{g}))$ is not Noetherian means that there are significant differences and difficulties in the representation theory of $\hat{L}(\mathfrak{g})$ and we shall see some of these in the rest of the notes.

2.4. Before defining the category $\hat{\mathcal{O}}$, we note the following result proved in $[22$ :

Proposition. The center of $\mathbf{U}(\hat{L}(\mathfrak{g}))$ is the polynomial algebra generated by the center $\mathbb{C} c$ of $\hat{L}(\mathfrak{g})$.

This proposition means that unlike Section 2, there is no good theory of central characters available for the study of representations of affine Lie algebras. However, many of the results discussed for $\mathcal{O}$ can be proved for $\hat{\mathcal{O}}$ and this is essentially because it is possible to define the analog of the Casimir operator. Recall that the definition given in (1.3) of the Casimir, only used dual bases with respect to the Killing form of $\mathfrak{g}$. This formula can be used verbatim for $\hat{L}(\mathfrak{g})$ by using the symmetric form defined above. However the sum is infinite and the Casimir operator lives in some completion of $\mathbf{U}(\hat{L}(\mathfrak{g}))$ and this means that it does not operate on all $\hat{L}(\mathfrak{g})$-modules. But it does act on modules in the category $\hat{\mathcal{O}}$ whose definition we now recall.

2.5. We say that an $\hat{L}(\mathfrak{g})$-module $M$ is an object of $\hat{\mathcal{O}}$ if it is a weight module, i.e., $\hat{\mathfrak{h}}$ acts semi-simply, $M=\oplus_{\mu \in \hat{\mathfrak{h}}^{*}} M_{\mu}$, and

$$
\operatorname{dim} M_{\mu}<\infty, \quad \text { wt } M \subset \bigcup_{s=1}^{p} \mu_{s}-\hat{Q}^{+},
$$

for some $p \in \mathbb{N}$ and elements $\mu_{1}, \cdots, \mu_{p} \in \hat{\mathfrak{h}}^{*}$. This definition differs from that of $\mathcal{O}$, in two ways. The first is that one does not require the module to be finitely generated. This is because one wants $\mathcal{O}$ to be closed under taking submodules and quotients and since $\mathbf{U}(\hat{L}(\mathfrak{g}))$ is not Noetherian a submodule of a finitely generated module need not be finitely generated. The condition that $\hat{\mathfrak{n}}^{+}$acts locally finitely on $M$ does not have all the implications it does in the category $\mathcal{O}$ (see Lemma 1.4) and hence is replaced by the stronger conditions in (2.1). Moreover, notice also that the second condition in (2.1) implies that if $\mu \in \mathrm{wt} M$, then $\mu+\beta \in$ wt $M$ only for finitely many $\beta \in \hat{\Phi}^{+}$. It is now immediate that the Casimir operator acts in a well-defined way on every $M \in \hat{\mathcal{O}}$ and moreover one can prove that it commutes with the action of $\hat{L}(\mathfrak{g})$.

2.6. For $\lambda \in \hat{\mathfrak{h}}^{*}$ we let $\hat{M}(\lambda)$ be the Verma module with highest weight $\lambda$ defined (with obvious modifications) in the same way as the Verma modules for simple Lie algebras given in Section 1.5. It has a unique irreducible quotient $\hat{V}(\lambda)$ and any irreducible module in $\hat{\mathcal{O}}$ is isomorphic to one of these and these results are proved in the same way as those for simple Lie algebras.

The following is a fairly simple exercise.

Lemma. Let $M \in \hat{\mathcal{O}}$ and assume that $\operatorname{dim} M<\infty$. Then $(L(\mathfrak{g}) \oplus \mathbb{C} c) M=0$. 
In other words, there are no interesting finite dimensional modules in $\hat{\mathcal{O}}$ and in view of Theorem [1.6 this raises the natural question, are irreducible modules associated to $\lambda \in \hat{P}^{+}$ distinguished in some way? The answer is yes and this leads us to the definition of an integrable module for an affine Lie algebra.

2.7. A weight module $\hat{V}$ for $\hat{L}(\mathfrak{g})$ is said to be integrable if the elements $x_{\alpha_{i}}^{ \pm}, 0 \leq i \leq n$ act locally nilpotently on $\hat{V}$. Integrable modules share many of the properties of finitedimensional $\mathfrak{g}$ modules. For instance a standard $\mathfrak{s l}_{2}$-argument shows that if $V$ is integrable then wt $V \subset \hat{P}$ and also that wt $V$ is $\hat{W}$-invariant. (Of course, one could have also defined integrable modules for $\mathfrak{g}$ but the definition is not interesting since one can prove that any integrable $\mathfrak{g}$-module is a sum of finite-dimensional $\mathfrak{g}$-modules). Let $\operatorname{ch} \hat{V}(\lambda)$ be the formal character of $\hat{V}(\lambda)$ as in (1.4). The following theorem explains the analogy between finitedimensional modules for $\mathfrak{g}$ and integrable modules in $\hat{\mathcal{O}}$.

Theorem. For $\lambda \in \hat{P}^{+}$, the module $\hat{V}(\lambda)$ is integrable and the full subcategory of $\hat{\mathcal{O}}$ consisting of integrable modules is completely reducible. Finally,

$$
\left(\sum_{w \in \hat{W}}(-1)^{\ell(w)} e(w \rho)\right) \operatorname{ch}(\hat{V}(\lambda))=\sum_{w \in \hat{W}}(-1)^{\ell(w)} e(w(\lambda+\rho)) .
$$

The formula (2.2) is called the Weyl-Kac character formula and the Casimir operator $\hat{\Omega}$ is an adequate substitute for the role played by the center in proving the Weyl character formula for a simple Lie algebra. The sums that appear in the Weyl-Kac character formula are infinite. This formula can be specialized in many ways and leads to interesting numbertheoretic identities, including combinatorial identities of Macdonald and the classical Jacobi triple product identity.

2.8. Suppose that $V$ is a weight module for $\hat{L}(\mathfrak{g})$, in which case we have

$$
V=\oplus_{a \in \mathbb{C}} V^{a}, \quad V^{a}=\{v \in V: c v=a v\},
$$

and $V^{a}$ is a $\hat{L}(\mathfrak{g})$-submodule. We say that $V^{a}$ is a module of level $a$.

Lemma. For $\lambda \in \hat{\mathfrak{h}}^{*}$, the module $\hat{V}(\lambda)$ has level $\lambda\left(h_{\theta}+h_{0}\right)$. Hence every irreducible integrable module in $\hat{\mathcal{O}}$ has non-negative integer level. The only level zero irreducible modules in $\hat{\mathcal{O}}$ are one-dimensional and correspond to taking $\lambda$ to be a scalar multiple of $\delta$.

Thus all the affine Lie algebras have a canonical integrable representation of level one corresponding to the weight $\Lambda_{0}$ and this is sometimes called the basic representation of $\hat{L}(\mathfrak{g})$. It is the simplest integrable representation in $\hat{\mathcal{O}}$ and the character formula given in Section 2.7 can be made explicit. The theory of vertex algebras, the relationship with physics and the connections with the monster group (see [9], 39], 40], [53, for instance) all have their roots in the effort to understand and explicitly construct the basic representation of the affine Lie algebra.

To conclude this section, we note that many of the problems studied for $\mathcal{O}$ have also been studied for $\hat{\mathcal{O}}$ and one does have a block decomposition [35] and a Kazhdan-Lusztig theory 
(see 47] for references to the literature on these topics). But the fact that the category $\hat{\mathcal{O}}$ is neither Noetherian nor Artinian does make things much more complicated. In the remaining sections our focus will be only on the integrable representations of affine Lie algebras. Finally, note that the results of this section are also known for the twisted affine algebras.

\section{Affine Lie algebras integrable Representations And Integral FORMS}

The reader has noticed by now that there are integrable representations of $\hat{L}(\mathfrak{g})$ which are not in $\hat{\mathcal{O}}$. The most obvious representation of a Lie algebra is the adjoint representation, and it is a simple matter to see from Section 2.2 that the adjoint representation of $\hat{L}(\mathfrak{g})$ is integrable but not in $\hat{\mathcal{O}}$. This raises the problem of classifying the irreducible integrable representations of affine Lie algebras, rather than just the ones in $\hat{\mathcal{O}}$. We address this problem in the first part of this section.

The adjoint representation is an example of a level zero representation which is indecomposable and reducible, since the center of $\hat{L}(\mathfrak{g})$ is a proper non-split submodule under the adjoint action. This shows that the category of level zero integrable representations of $\hat{L}(\mathfrak{g})$ is not semisimple and hence should have interesting homological properties. To study these properties and to pursue the directions of study which have been fruitful in the case of simple Lie algebras, it is helpful to understand (following H. Garland) the integral form of the universal enveloping algebra of $\hat{L}(\mathfrak{g})$. Garland also proved that the integrable modules in $\hat{\mathcal{O}}$ have an integral form, but relatively little is known about the corresponding representations of the hyper-algebra, except in the case of the basic representation which we discuss briefly.

3.1. We begin by making some comments about duality. Let $w_{0} \in W$ be the longest element of the Weyl group of $\mathfrak{g}$. It is well-known that the dual of an irreducible finitedimensional representation of $\mathfrak{g}$ with highest weight $\lambda$ is a highest weight representation with highest weight $-w_{0} \lambda$. Let us now consider the situation of irreducible integrable modules in $\hat{\mathcal{O}}$. The first difficulty one encounters, is that the module $\hat{V}(\lambda)$ is infinite-dimensional and the dual space is too big. The weight spaces, however, are finite-dimensional and so one works instead with the restricted dual

$$
\hat{V}(\lambda)^{\#}=\bigoplus_{\mu \in \hat{P}^{+}} V(\lambda)_{\mu}^{*}
$$

which is an integrable $\hat{L}(\mathfrak{g})$-module. Since $\hat{W}$ is an infinite group, one does not have the analog of the longest element: there does not exist $w \in \hat{W}$ such that $w \hat{\Pi}=-\hat{\Pi}$ and this means that $\hat{V}(\lambda)^{\#}$ is not a highest weight module. However, it is easily seen that it is a lowest weight module, which is defined in the obvious way, by replacing $\hat{\mathfrak{n}}^{+}$by $\hat{\mathfrak{n}}^{-}$. The lowest weight space is $V(\lambda)_{\lambda}^{*}$ and the lowest weight is $-\lambda$. But apart from this modification, it is clear that understanding highest weight modules is the same as understanding lowest weight modules.

3.2. The category of all integrable modules for $\hat{L}(\mathfrak{g})$ is very big, even if we restrict to a fixed level. To make the study more manageable we let $\mathcal{I}$ be the category whose objects are $\hat{L}(\mathfrak{g})$-modules with finite-dimensional weight spaces. Our goal is to classify the irreducible objects in $\mathcal{I}$, and in the previous section we saw how to construct irreducible objects of $\mathcal{I}$ which are also in $\hat{\mathcal{O}}$. But so far we have only encountered one interesting example (see Lemma 
2.8) of a level zero representation, the adjoint, and that was reducible. Our first task then, is to construct natural examples of irreducible representations of level zero.

Given a representation $V$ of $\mathfrak{g}$, set $L(V)=V \otimes \mathbb{C}\left[t, t^{-1}\right]$. For $a \in \mathbb{C}^{\times}$, one can define the structure of a $\hat{L}(\mathfrak{g})$-module on $L(V)$ by:

$$
\left(x \otimes t^{r}\right)\left(v \otimes t^{s}\right)=a^{r} x v \otimes t^{r+s}, \quad c(L(V))=0, \quad d\left(v \otimes t^{r}\right)=r v \otimes t^{r} .
$$

We denote this module by $L\left(\operatorname{ev}_{a}^{*} V\right)$ and we will say more about this notation later. This construction can be generalized further as follows. Suppose that we are given representations $\left\{V_{s}: 1 \leq s \leq k\right\}$ of $\mathfrak{g}$ and $k$ non-zero complex numbers $a_{1}, \cdots, a_{k}$. The space $L\left(V_{1} \otimes \cdots \otimes V_{k}\right)$ has a $\hat{L}(\mathfrak{g})$-module structure defined by

$$
\begin{gathered}
\left(x \otimes t^{r}\right)\left(v_{1} \otimes \cdots \otimes v_{k} \otimes t^{m}\right)=\sum_{s=1}^{k}\left(v_{1} \otimes \cdots \otimes v_{s-1} \otimes x v_{s} \otimes v_{s+1} \otimes \cdots \otimes v_{k} \otimes a_{s}^{r} t^{r+m}\right), \\
c L\left(V_{1} \otimes \cdots \otimes V_{k}\right)=0, \\
d\left(v_{1} \otimes \cdots \otimes v_{k} \otimes t^{m}\right)=m\left(v_{1} \otimes \cdots \otimes v_{k} \otimes t^{m}\right),
\end{gathered}
$$

and we denote this module by $L\left(\operatorname{ev}_{a_{1}}^{*} V_{1} \otimes \cdots \otimes \mathrm{ev}_{a_{k}}^{*} V_{k}\right)$. Modules of these kind are sometimes called loop modules. The following result was proved in [29].

Proposition. Let $k \in \mathbb{N}$, and for $1 \leq s \leq k$ let $\lambda_{s} \in P^{+}$and $a_{s} \in \mathbb{C}^{\times}$and assume that $a_{s} \neq a_{m}$ if $s \neq m$. There exists $r \geq 1$ such that

$$
\sum_{s=1}^{k} \lambda_{s}(h) a_{s}^{j}=0, \quad 1 \leq j<r, \text { for all } h \in \mathfrak{h} .
$$

For $1 \leq j \leq r$, the submodule

$$
L^{j}\left(\mathrm{ev}_{a_{1}}^{*} V\left(\lambda_{1}\right) \otimes \cdots \otimes \mathrm{ev}_{a_{k}}^{*} V\left(\lambda_{k}\right)\right)=\mathbf{U}(\hat{L}(\mathfrak{g}))\left(v_{\lambda_{1}} \otimes \cdots \otimes v_{\lambda_{k}} \otimes t^{j}\right)
$$

is irreducible and we have an isomorphism of $\hat{L}(\mathfrak{g})$-modules,

$$
L\left(\operatorname{ev}_{a_{1}}^{*} V\left(\lambda_{1}\right) \otimes \cdots \otimes \mathrm{ev}_{a_{k}}^{*} V\left(\lambda_{k}\right)\right) \cong \bigoplus_{s=1}^{r} L^{s}\left(\operatorname{ev}_{a_{1}}^{*} V\left(\lambda_{1}\right) \otimes \cdots \otimes \operatorname{ev}_{a_{k}}^{*} V\left(\lambda_{k}\right)\right)
$$

The statement in [29] is more precise and gives the value of $r$ for a fixed set of $\lambda_{s}, a_{s}$, $1 \leq s \leq k$. As an example, one has $r=2$ when $\lambda_{1}=\lambda_{2}$ and $a_{1}=-a_{2}$.

3.3. The following is an amalgamation of results proved in [14] and [29] and achieves our goal of classifying the irreducible objects of $\mathcal{I}$. Recall that if $V$ is integrable, then wt $V \subset \hat{P}$ and hence the center $c=h_{0}+h_{\theta}$ acts on $V$ with integer eigenvalues.

Theorem. Let $V \in \mathrm{Ob} \mathcal{I}$ be irreducible and let $k \in \mathbb{Z}$ be such that $c v=k v$ for all $v \in V$.

(i) If $k>0$ then $V \in \hat{\mathcal{O}}$ and hence $V \cong \hat{V}(\lambda)$ for some $\lambda \in \hat{P}^{+}$.

(ii) If $k<0$ then $V \cong \hat{V}(\lambda)$ \# for some $\lambda \in \hat{P}^{+}$. 
(iii) If $k=0$, then

$$
V \cong L^{s}\left(\mathrm{ev}_{a_{1}}^{*} V\left(\lambda_{1}\right) \otimes \cdots \otimes \mathrm{ev}_{a_{k}}^{*} V\left(\lambda_{k}\right)\right),
$$

for some $\lambda_{1}, \cdots, \lambda_{k} \in P^{+}, a_{1}, \cdots, a_{k} \in \mathbb{C}^{\times}$and $s$ as in Proposition 3.2 .

The twisted version of this theorem is known and may be found in [30].

We now make some remarks which will allow us to limit our focus in the next sections on finite-dimensional representations of $L(\mathfrak{g})$. The first observation is that to produce a representation of $\hat{L}(\mathfrak{g})$ from a representation of $\mathfrak{g}$ one has to tensor with $\mathbb{C}\left[t, t^{-1}\right]$ only to keep track of the grading that $d$ defines on $\hat{L}(\mathfrak{g})$. But one can get around this problem by defining a functor $\mathbf{L}$ from the category of $L(\mathfrak{g})$-modules to $\hat{L}(\mathfrak{g})$-modules, which is given on objects by,

$$
\begin{gathered}
\mathbf{L}(V)=V \otimes \mathbb{C}\left[t, t^{-1}\right], \\
\left(x \otimes t^{r}\right)\left(v \otimes t^{s}\right)=\left(x \otimes t^{r} v\right) \otimes t^{r+s}, \quad c \mathbf{L}(V)=0, \quad d\left(v \otimes t^{r}\right)=r v \otimes t^{r} .
\end{gathered}
$$

Clearly $\mathbf{L}$ takes finite-dimensional $L(\mathfrak{g})$-modules to integrable $\hat{L}(\mathfrak{g})$-modules. It will become clear from the results of the next section, that the functor $\mathbf{L}$ in general sends irreducible finite-dimensional modules to completely reducible integrable ones. In [18] it is shown that $\mathbf{L}$ preserves enough of the homological properties of $L(\mathfrak{g})$-modules to make it worthwhile to restrict one's attention to modules for $L(\mathfrak{g})$.

3.4. We digress, briefly, from our study of $\mathcal{I}$ to define the imaginary integral root vectors and the integral form of $\mathbf{U}(\hat{L}(\mathfrak{g}))$. These were introduced by Garland in 43. and allow us to study lattices in integrable modules and hence also the representations of the hyperalgebra of $\hat{L}(\mathfrak{g})$. It is also worthwhile to note that the integral imaginary root vectors play a major role in the vertex operator construction [38, of the basic representation which was defined in Section 2.8 ,

As in the case of $\mathfrak{g}$ we let $\mathbf{U}_{\mathbb{Z}}(\hat{L}(\mathfrak{g}))$ be the $\mathbb{Z}$-subalgebra of $\mathbf{U}(\hat{L}(\mathfrak{g}))$ generated by the elements $\left(x_{\alpha}^{ \pm} \otimes t^{s}\right)^{(r)}$, where $\alpha \in \Phi^{+}, s \in \mathbb{Z}$ and $r \in \mathbb{N}$. We know from Section 1.11 that one has to be more careful with the Cartan type elements $\left(h_{i} \otimes t^{s}\right), s \in \mathbb{Z}$. For $s=0, r \in \mathbb{N}$ and $1 \leq i \leq n$, the elements $\left(\begin{array}{c}h_{i} \\ r\end{array}\right)$ are in $\mathbf{U}_{\mathbb{Z}}(\mathfrak{g})$ and hence in $\mathbf{U}_{\mathbb{Z}}(\hat{L}(\mathfrak{g}))$.

To define the analogs of the divided powers for the imaginary root vectors $h_{i} \otimes t^{s}, i \in I$, $s \in \mathbb{Z}, s \neq 0$ one tries to modify suitably the principle we used in Section 1.11 (see equation (1.10) $)$. Consider the triangular decomposition

$$
\mathbf{U}(\hat{L}(\mathfrak{g}))=\mathbf{U}\left(\hat{\mathfrak{n}}^{-}\right) \mathbf{U}(\hat{\mathfrak{h}}) \mathbf{U}\left(\hat{\mathfrak{n}}^{+}\right),
$$

and note that for all $\alpha \in \Phi^{+}, r, m \in \mathbb{N}$ the element $\left(x_{\alpha}^{+} \otimes t\right)^{(r)}\left(x_{\alpha}^{-}\right)^{(m)}$, is not in the order prescribed on the right hand side. In [43, Garland proved a remarkable formula which rewrites this element in the correct order. We will not reproduce his entire formula here, but content ourselves with writing down the correct analog (which emerges from his formula) of the divided power of the imaginary root vectors. For each $i \in I$ we define formal power series $P_{i}^{ \pm}(u)$ in an indeterminate $u$ with values in the commutative algebra $\mathbf{U}\left(\mathfrak{h} \otimes t^{ \pm 1} \mathbb{C}\left[t^{ \pm 1}\right]\right)$ by

$$
P_{i}^{ \pm}(u)=\sum_{s=0}^{\infty} P_{i, s}^{ \pm} u^{s}=\exp \left(-\sum_{k=1}^{\infty} \frac{h_{i} \otimes t^{ \pm k}}{k}\right),
$$


and we can now state Garland's theorem.

Theorem. Fix an order on the set

$$
\left\{\left(x_{\alpha}^{ \pm} \otimes t^{s}\right)^{(r)}: \alpha \in \Phi^{+}, s \in \mathbb{Z}, r \in \mathbb{N}\right\} \cup\left\{P_{i, s}^{ \pm}: 1 \leq i \leq n, s \in \mathbb{N}\right\} \cup\left\{\left(\begin{array}{c}
h_{i} \\
r
\end{array}\right): i \in I, r \in \mathbb{N}\right\} .
$$

The ordered monomials from this set are a $\mathbb{Z}$-basis of $\mathbf{U}_{\mathbb{Z}}(\hat{L}(\mathfrak{g}))$ and a $\mathbb{C}$-basis of $\mathbf{U}(\hat{L}(\mathfrak{g}))$. Moreover, if $\lambda \in \hat{P}^{+}$and $\hat{v}_{\lambda} \in \hat{V}(\lambda)_{\lambda}$, then $\mathbf{U}_{\mathbb{Z}}(\hat{L}(\mathfrak{g})) \hat{v}_{\lambda}$ is a $\mathbb{Z}$-lattice in $\hat{V}(\lambda)$.

The twisted version of this theorem may be found in [62].

3.5. In view of this theorem it would be natural to pass to a field $\mathbb{F}$ of characteristic $p$ as we did in Section 1.11 and study the representations $\mathbf{U}(\hat{L}(\mathfrak{g}))_{\mathbb{F}}$ and in particular the modules $\hat{V}(\lambda)_{\mathbb{F}}$. However, this direction does not seem to have been pursued and except for the result we now discuss, virtually nothing is known about the irreducible modules of positive level in characteristic $p$. In [23], a quantum analog of this problem was studied and it was shown there that the basic representation of the quantum affine algebra remained irreducible at an $m^{t h}$ root of unity, where $m$ is odd and coprime to the determinant of the Cartan matrix of $\mathfrak{g}$. This was proved essentially by constructing the basic representation at a root of unity and showing that it had the same character. The proof given in that paper works verbatim in the characteristic $p$ case as long as $p$ is odd and coprime to the determinant of the Cartan matrix of $\mathfrak{g}$. The case of level zero modules in characteristic $p$ has been studied by Jakelic and Moura in [49], [50], [51] and we shall discuss this in the next sections.

\section{Finite-Dimensional modules for loop Algebras AND their GENERALIZATIONS}

In this section, we discuss the category of finite-dimensional representations of loop algebras, study extensions between irreducible modules and describe the blocks of this category.

4.1. For $a \in \mathbb{C}^{\times}$let $\mathrm{ev}_{a}: L(\mathfrak{g}) \rightarrow \mathfrak{g}$ be the homomorphism of Lie algebras given by

$$
\mathrm{ev}_{a}(x \otimes f)=f(a) x \text {. }
$$

If $V$ is a $\mathfrak{g}$-module, we denote the corresponding $L(\mathfrak{g})$-module by $\operatorname{ev}_{a}^{*} V$. Together with the discussion in Section [3.3, this explains the notation $L\left(\operatorname{ev}_{a}^{*} V\right)$ used in Section 3.2. The following result classifies irreducible finite-dimensional representations of $L(\mathfrak{g})$.

Theorem. An irreducible finite-dimensional representation of $L(\mathfrak{g})$ is isomorphic to a tensor product $\mathrm{ev}_{a_{1}}^{*} V\left(\lambda_{1}\right) \otimes \cdots \otimes \mathrm{ev}_{a_{k}}^{*} V\left(\lambda_{k}\right)$, where $\lambda_{s} \in P^{+}, a_{s} \in \mathbb{C}^{\times}$with $a_{s} \neq a_{r}$ for $1 \leq s, r \leq k$. Conversely any such tensor product is irreducible.

This result can be deduced easily from Proposition 3.2 and Theorem 3.3(iii). A more direct proof was given by Rao in [67] along the following lines. If $\rho: L(\mathfrak{g}) \rightarrow$ End $V$ is a finitedimensional representation, then the kernel of $\rho$ is an ideal of finite-codimension in $L(\mathfrak{g})$. It is not too hard to show that any ideal in $L(\mathfrak{g})$ must be of the form $\mathfrak{g} \otimes I(V)$ where $I(V)$ is an ideal in $\mathbb{C}\left[t, t^{-1}\right]$. If $V$ is irreducible then one proves that $I(V)$ is a product of distinct maximal ideals and hence is generated by an element $\left(t-a_{1}\right) \cdots\left(t-a_{k}\right)$ for some $k \in \mathbb{N}$ and distinct elements $a_{1}, \cdots, a_{k}$ in $\mathbb{C}^{\times}$Moreover $\mathbb{C}\left[t, t^{-1}\right] / I(V)$ is a vector space of dimension $k$ and it follows now that $L(\mathfrak{g}) /(\mathfrak{g} \otimes I(V))$ is isomorphic to a direct sum of $k$-copies of $\mathfrak{g}$ 
and hence is a semisimple Lie algebra. The theorem now follows by using the representation theory of semisimple Lie algebras.

For the twisted affine Lie algebras the corresponding result was proved in [15]. To describe it, note that since $L(\mathfrak{g}, \sigma, m)$ is a subalgebra of $L(\mathfrak{g})$, one can regard the tensor product $V=\mathrm{ev}_{a_{1}}^{*} V\left(\lambda_{1}\right) \otimes \cdots \otimes \mathrm{ev}_{a_{k}}^{*} V\left(\lambda_{k}\right)$ as a module for the twisted algebra. In general it is not irreducible as a module for the twisted algebra, but if one imposes the additional condition that $a_{s}^{m} \neq a_{r}^{m}$ for all $1 \leq s, r \leq k$, where $m$ is the order of $\sigma$, then $V$ is an irreducible module for $L(\mathfrak{g}, \sigma, m)$. Moreover, one can also prove that these are exactly (up to isomorphism) all the finite-dimensional irreducible modules.

4.2. An element $a \in \mathbb{C}^{\times}$determines a maximal ideal $(t-a)$ of $\mathbb{C}\left[t, t^{-1}\right]$ and another way to formulate Theorem 4.1 is to say that the irreducible finite-dimensional representations are parametrized by finitely-supported functions from the maximal spectrum of $\mathbb{C}\left[t, t^{-1}\right]$ to $P^{+}$, where by finitely supported, we mean $\chi(M)=0$ for all but finitely many maximal ideals. We generalize the theorem to the case of $\mathfrak{g} \otimes A$, where $A$ is a finitely generated commutative associative algebra algebra over $\mathbb{C}$. The space $\mathfrak{g} \otimes A$ is a Lie algebra as usual: $[x \otimes a, y \otimes b]=[x, y] \otimes a b$. Let $\max A$ be the set of maximal ideals in $A$ and denote by $\Xi\left(\max A, P^{+}\right)$the set of finitely supported functions from $\max A$ to $P^{+}$, and set

$$
\operatorname{supp} \psi=\{M \in \max A: \psi(M) \neq 0\}, \quad \psi \in \Xi\left(\max A, P^{+}\right) .
$$

For $M \in \max A$, let $\operatorname{ev}_{M}: \mathfrak{g} \otimes A \rightarrow \mathfrak{g}$ be the map of Lie algebras which is induced by the algebra homomorphism $A \rightarrow A / M \cong \mathbb{C}$ and we denote by $\operatorname{ev}_{M}^{*} V(\lambda)$ the representation of $\mathfrak{g} \otimes A$ obtained by pulling back $V(\lambda)$ through $\mathrm{ev}_{M}$.

Theorem. Let $A$ be a finitely generated commutative and associative algebra over $\mathbb{C}$. For $\chi \in \Xi\left(\max A, P^{+}\right)$, the $\mathfrak{g} \otimes A$-module $\bigotimes_{M \in \operatorname{supp} \chi} \operatorname{ev}_{M}^{*} V(\chi(M))$ is irreducible and conversely any finite dimensional irreducible representation is isomorphic to one of these.

The theorem has been proved by many people in various cases: in the case when $A$ is the polynomial ring in $r$ variables the result was first proved by Rao [67], the general case given above appears in [16]. In [59], Lau also obtains a proof of this statement for the Laurent polynomial ring in $r$ variables and he is also able prove the result in the twisted case. It is important to note here, that unlike in the case when $A=\mathbb{C}\left[t, t^{-1}\right]$, the twisted algebras are not determined by a Dynkin diagram automorphism and one has many non-isomorphic twisted algebras and Lau works in this generality. The methods of all these papers are algebraic and similar to the proof sketched above for the loop algebra. A more geometric approach is developed in [66] and their methods allow them to develop a uniform approach which works for twisted and untwisted algebras of the form $\mathfrak{a} \otimes A$ where $\mathfrak{a}$ is an arbitrary Lie algebra. In this generality the irreducible representations are not always tensor products of evaluations, but they are very nearly so, up to tensoring with a one-dimensional representation.

4.3. An obvious and not very difficult question that one should ask is what kind of module one gets if one allows $a_{r}=a_{s}$ for some pair $1 \leq r, s, \leq k$ in the statement of Theorem 4.1. The following proposition proved in [17] shows that the module is completely reducible and more generally gives a necessary and sufficient condition for a finite-dimensional module to be completely reducible. Recall that for any $L(\mathfrak{g})$-module $V$ we have defined in Section 
4.1 an ideal $I(V)$ of $\mathbb{C}\left[t, t^{-1}\right]$ which is maximal with the property that $(\mathfrak{g} \otimes I(V)) V=0$. More precisely, $\mathfrak{g} \otimes I(V)$ is the annihilating ideal of $V$ in $L(\mathfrak{g})$.

Proposition. Let $\lambda, \mu \in P^{+}$and $a \in \mathbb{C}^{\times}$. Then $\mathrm{ev}_{a}^{*} V(\lambda) \otimes \mathrm{ev}_{a}^{*} V(\mu)$ is isomorphic to a direct sum of submodules of the form $\operatorname{ev}_{a}^{*} V(\nu), \nu \in P^{+}$and

$$
\operatorname{Hom}_{L(\mathfrak{g})}\left(\operatorname{ev}_{a}^{*} V(\nu), \mathrm{ev}_{a}^{*} V(\lambda) \otimes \mathrm{ev}_{a}^{*} V(\mu)\right) \cong \operatorname{Hom}_{\mathfrak{g}}(V(\nu), V(\lambda) \otimes V(\mu)) .
$$

More generally, a finite-dimensional module $V$ is completely reducible iff the ideal $I(V)$ is a product of distinct maximal ideals. Analogous statements hold for algebras of type $\mathfrak{g} \otimes A$.

4.4. We now describe extensions between irreducible $L(\mathfrak{g})$-modules. We begin with a simple case and compute $\operatorname{Ext}_{L(\mathfrak{g})}^{1}\left(\operatorname{ev}_{a}^{*} V(\lambda), \operatorname{ev}_{a}^{*} V(\mu)\right)$ for $a \in \mathbb{C}^{\times}$.

Let

$$
0 \rightarrow \mathrm{ev}_{a}^{*} V(\mu) \rightarrow U \rightarrow \mathrm{ev}_{a}^{*} V(\lambda) \rightarrow 0
$$

be a short exact sequence of $L(\mathfrak{g})$-modules. Regarded as a short exact sequence of $\mathfrak{g}$-modules this sequence is split and we can pick $u_{\lambda} \in U_{\lambda}$ with $\mathbf{U}(\mathfrak{g}) u \cong V(\lambda)$. If (4.1) is not split as a short exact sequence of $L(\mathfrak{g})$-modules, there must exist $r \in \mathbb{Z}$ such that the $\mathfrak{g}$-module map,

$$
\mathfrak{g} \otimes \mathbf{U}(\mathfrak{g}) u_{\lambda} \rightarrow U, \quad\left(x t^{r} \otimes g u_{\lambda}\right) \rightarrow x t^{r} g u_{\lambda},
$$

has a non-zero projection (as a $\mathfrak{g}$-module) onto the image of $\operatorname{ev}_{a}^{*} V(\mu)$, i.e.,

$$
\operatorname{Ext}_{L(\mathfrak{g})}^{1}\left(\mathrm{ev}_{a}^{*} V(\lambda), \mathrm{ev}_{a}^{*} V(\mu)\right) \neq 0 \Longrightarrow \operatorname{Hom}_{\mathfrak{g}}(\mathfrak{g} \otimes V(\lambda), V(\mu)) \neq 0 .
$$

Conversely, given $\pi: \mathfrak{g} \otimes V(\lambda) \rightarrow V(\mu)$ a map of $\mathfrak{g}$-modules it is proved in 27] that the formula

$$
x t^{r}(v, w)=\left(a^{r} x v, a^{r} x w+r a^{r-1} \pi(x v)\right), \quad v \in V(\lambda), w \in V(\mu), \quad a \in \mathbb{C}^{\times}, \quad r \in \mathbb{Z}, \quad x \in \mathfrak{g},
$$

defines an indecomposable $L(\mathfrak{g})$-module denoted $V(\lambda, \mu, a)$ on $V(\lambda) \oplus V(\mu)$ and hence one can conclude that

$$
\operatorname{Ext}_{L(\mathfrak{g})}^{1}\left(\mathrm{ev}_{a}^{*} V(\lambda), \mathrm{ev}_{a}^{*} V(\mu)\right) \cong \operatorname{Hom}_{\mathfrak{g}}(\mathfrak{g} \otimes V(\lambda), V(\mu)),
$$

which can be shown to be equivalent to

$$
\operatorname{Ext}_{L(\mathfrak{g})}^{1}\left(\mathrm{ev}_{a}^{*} V(\lambda), \mathrm{ev}_{a}^{*} V(\mu)\right) \cong \operatorname{Hom}_{L(\mathfrak{g})}\left(\mathrm{ev}_{a}^{*} \mathfrak{g} \otimes \mathrm{ev}_{a}^{*} V(\lambda), \quad \mathrm{ev}_{a}^{*} V(\mu)\right) .
$$

The general statement proved in [17] (for $\mathfrak{g} \otimes \mathbb{C}[t]$ but which has an obvious modification to $L(\mathfrak{g}))$ is:

Theorem. Let $V$ and $V^{\prime}$ be irreducible finite-dimensional $L(\mathfrak{g})$-modules. Then

$$
\operatorname{dim} \operatorname{Ext}_{L(\mathfrak{g})}^{1}\left(V, V^{\prime}\right)=\sum_{a \in \mathbb{C}^{\times}} \operatorname{dim} \operatorname{Hom}_{L(\mathfrak{g})}\left(\operatorname{ev}_{a}^{*} \mathfrak{g} \otimes V, V^{\prime}\right) .
$$

This condition can be made quite explicit using the results stated above, namely if we write $V$ and $V^{\prime}$ as a tensor product of evaluation representations as in Theorem 4.1 one can compute the dimension of $\operatorname{Ext}_{L(\mathfrak{g})}^{1}\left(V, V^{\prime}\right)$ as the sum of multiplicities of the adjoint representation of $\mathfrak{g}$ in a tensor product of the form $V(\lambda) \otimes V(\mu)^{*}$ for suitable $\lambda, \mu$. 
4.5. The analogous result has not been established as yet for the twisted Lie algebras. It has been solved in [56] for the more general Lie algebra $\mathfrak{g} \otimes A$ and in fact the main theorem in this paper is written in a very explicit way. There is one new feature though which is not seen in the case of $\mathbb{C}\left[t, t^{-1}\right]$ which we explain again in a simple case. For $\lambda, \mu \in P^{+}$and $M \in \max A$, we have

$$
\operatorname{Ext}_{\mathfrak{g} \otimes A}^{1}\left(\mathrm{ev}_{M}^{*} V(\lambda), \mathrm{ev}_{M}^{*} V(\mu)\right) \cong \operatorname{Hom}_{\mathfrak{g}}(\mathfrak{g} \otimes V(\lambda), V(\mu)) \otimes \operatorname{Der}(A, A / M),
$$

where $\operatorname{Der}(A, A / M)$ is the space of all linear maps $A \rightarrow A / M$ satisfying $D(a b)=a D(b)+$ $b D(a)$. In the case when $A=\mathbb{C}\left[t, t^{-1}\right]$ this space is one-dimensional and so is invisible.

4.6. To continue our study of finite-dimensional representations, we look back at Section 2 and notice that one of the natural things to do is to understand the blocks of the category. As we already noted in Section 4, the theory of central characters is not available for affine or loop algebras. However, it is still possible to describe the blocks and to do this one uses a family of universal indecomposable finite-dimensional modules introduced in [33] and called the Weyl modules for affine Lie algebras. These are not the same modules as the ones discussed in Section 2, but are in some sense obtained in the same way. They can be regarded as the classical limit of irreducible representations of quantum affine algebras and we shall say more about this later. The first step is to organize the irreducible representations in some nice way and for this, we recall from [27] the notion of the spectral character of a $L(\mathfrak{g})$-module.

Definition. Given an irreducible representation $V=\otimes_{s=1}^{k} \operatorname{ev}_{a_{s}}^{*} V\left(\lambda_{s}\right)$ of $L(\mathfrak{g})$ the spectral character of $V$ is defined to be the function $\chi_{V}: \mathbb{C}^{\times} \rightarrow P / Q$ given by

$$
\chi_{V}(z)=\left\{\begin{array}{l}
0, \quad z \notin\left\{a_{1}, \cdots, a_{k}\right\}, \\
\lambda_{s}+Q, \quad z=a_{s}, \quad 1 \leq s \leq k .
\end{array}\right.
$$

A finite dimensional $L(\mathfrak{g})$-module is said to have spectral character if all the irreducible modules occurring in a Jordan-Hölder series have the same spectral character.

Note that it is very easy for two modules to have the same spectral character, for instance if $\lambda, \mu \in P^{+} \cap Q$, then

$$
\chi_{\operatorname{ev}_{a}^{*} V(\lambda)}=\chi_{\operatorname{ev}_{b}^{*} V(\mu)}, \text { for all } a, b \in \mathbb{C}^{\times} .
$$

4.7. We recall the definition of the blocks of a category $\mathcal{C}$.

Definition. Say that two indecomposable objects $U, V \in \mathrm{ObC}$ are linked if there do not exist abelian subcategories $\mathcal{C}_{k}, k=1,2$ such that $\mathcal{C}=\mathcal{C}_{1} \oplus \mathcal{C}_{2}$ with $U \in \mathcal{C}_{1}$ and $V \in \mathcal{C}_{2}$. If $U$ and $V$ are decomposable then we say that they are linked if every indecomposable summand of $U$ is linked to every indecomposable summand of $V$. This defines an equivalence relation on $\mathcal{C}$ and the equivalence classes are called the blocks of $\mathcal{C}$.

The main result proved in [27] is the following theorem which describes the blocks of the category of finite-dimensional representations.

Theorem. Let $V$ be a finite-dimensional $L(\mathfrak{g})$ module. Then $V$ is isomorphic to a direct sum of finite-dimensional modules $V_{r}, 1 \leq r \leq k$ each of which admits a spectral character. Moreover, any two modules with the same spectral character are linked. 
As a consequence of this theorem, we see that the blocks are very large subcategories, for instance when $\mathfrak{g}$ is of type $E_{8}$ there is only one block, i.e., any two finite-dimensional modules are linked. A similar result was proved in [69] for the twisted algebras $L(\mathfrak{g}, \sigma, m)$ and in [56] for the Lie algebras $\mathfrak{g} \otimes A$. All the proofs are similar and involve two ingredients. The first is the module $V(\lambda, \mu, a)$ constructed in Section 4.4 and the second are the Weyl modules which we discuss in the next section.

4.8. Most of the results of this section have analogs in positive characteristic for the hyperalgebras $\mathbf{U}(L(\mathfrak{g}))_{\mathbb{F}}$ and these are studied in [49], [50]. The one exception is the result on extensions between irreducible modules. This is because the proof given in characteristic zero relies on the fact that a finite-dimensional representation of a complex simple Lie algebras is completely reducible, which as we remarked in Section 1.11 is false in positive characteristic.

\section{Weyl modules, RESTRICTED KIRILlOV-REshetikHin AND BEyOND}

We begin this section by discussing briefly the input coming from the representation theory of quantum affine algebras, which has helped to identify interesting families of finitedimensional representations of loop algebras. The interested reader could consult [21] and the references therein for further details on representations of quantum affine algebras.

5.1. In the 1980's Drinfeld and Jimbo introduced the quantized enveloping algebras $\mathbf{U}_{q}(\mathfrak{a})$ of a Kac-Moody Lie algebra $\mathfrak{a}$. These algebras depend on a parameter $q$ which can be either a complex number, in which case one regards $\mathbf{U}_{q}(\mathfrak{a})$ as an algebra over $\mathbb{C}$ or a formal variable in which case one regards it as an algebra over the function field $\mathbb{C}(q)$, and informally speaking if we put $q=1$ we get the usual enveloping algebra of $\mathfrak{a}$. In the case when $\mathfrak{g}$ is simple, Lusztig [?] proved that for generic $q$, the irreducible finite-dimensional representations of $\mathbf{U}_{q}(\mathfrak{g})$ are given by elements of $P^{+}$and moreover that the module associated to $\lambda \in P^{+}$has the same character (suitably understood) as the module in the $q=1$ case, namely it is the character of the $\mathfrak{g}$-module $V(\lambda)$. An analogous statement was also proved for the positive level integrable modules of the quantized affine algebras. In the case when $q$ is a primitive $m^{t h}$-root of unity, the picture resembles the case of positive characteristic and the irreducible modules are in general smaller.

5.2. In the case of finite-dimensional modules for the quantized affine algebras the difference between generic $q$ and the roots of unity case is already visible when $q=1$. In other words the action of $L(\mathfrak{g})$ on evaluation representations and their tensor products given in Theorem 4.1 cannot be deformed to give an action of the quantum affine algebra on the same space. Or, to put it in yet another way, the $q=1$ limit of irreducible representations of quantum affine algebras generally give rise to a reducible indecomposable representation of $L(\mathfrak{g})$. This was the motivation for introducing in [33] the definition of Weyl modules for affine Lie algebras. We should remark here that this phenomenon was first noted in [36] in connection with the representation theory of the closely related Yangians, which are deformations of the current algebra, $\mathfrak{g} \otimes \mathbb{C}[t]$. 
5.3. The classification of irreducible finite-dimensional representations for quantum affine algebras was obtained in 31 and is similar to the one obtained by Drinfeld for the Yangians. It was also very similar to the abstract classification (rather than the more explicit one described in these notes) of loop modules given in [14]. However, the fact that there is no analog of the evaluation homomorphism from the quantum loop algebra to the quantum simple algebra has meant that describing the irreducible modules in a concrete way has been challenging and a variety of methods have been developed to understand these modules better. One of these methods is to understand the multiplicity with which an irreducible module for the quantum simple algebra occurs in an irreducible module for the quantum loop algebra. This can be turned into a problem of the non-quantum case for the following reason. It was proved in 33 that under natural conditions the irreducible representations of the quantum affine algebra admitted a $\mathbb{C}\left[t, t^{-1}\right]$-form and hence could be specialized to $q=1$ and gives a representation of $L(\mathfrak{g})$. A result of Lusztig implies that understanding the $\mathfrak{g}$-module decomposition of this module is the same as answering the question for the quantum case. With this motivation out of the way, we now return our focus to representations of $L(\mathfrak{g})$.

5.4. For $\lambda \in P^{+}$, let $W(\lambda)$ be the $L(\mathfrak{g})$-module generated by an element $w_{\lambda}$ with relations

$$
h w_{\lambda}=\lambda(h) w_{\lambda}, \quad L\left(\mathfrak{n}^{+}\right) w_{\lambda}=0, \quad\left(x_{\alpha_{i}}^{-}\right)^{\lambda\left(h_{\alpha_{i}}\right)+1} w_{\lambda}=0 .
$$

We call $W(\lambda)$ the global Weyl module. The following is elementary.

Lemma. The module $W(\lambda)$ is integrable for all $\lambda \in P^{+}$. Moreover if $V$ is any integrable module, then

$$
\operatorname{Hom}_{L(\mathfrak{g})}(W(\lambda), V) \cong V_{\lambda}^{+}, \quad V_{\lambda}^{+}=\left\{v \in V_{\lambda}: L\left(\mathfrak{n}^{+}\right) v=0\right\} .
$$

In particular, if $\mathrm{ev}_{a_{1}}^{*} V\left(\lambda_{1}\right) \otimes \cdots \otimes \mathrm{ev}_{a_{k}}^{*} V\left(\lambda_{k}\right)$ is an irreducible finite-dimensional $L(\mathfrak{g})$-module with $\sum_{s=1}^{k} \lambda_{s}=\lambda$, then

$$
\operatorname{dim} \operatorname{Hom}_{L(\mathfrak{g})}\left(W(\lambda), \mathrm{ev}_{a_{1}}^{*} V\left(\lambda_{1}\right) \otimes \cdots \otimes \mathrm{ev}_{a_{k}}^{*} V\left(\lambda_{k}\right)\right)=1 .
$$

The lemma shows immediately that the module $W(\lambda)$ is infinite-dimensional if $\lambda \neq 0$ and in fact that $W(\lambda)_{\lambda}$ is infinite-dimensional. For otherwise, the action of the algebra $L(\mathfrak{h})$ on $W(\lambda)_{\lambda}$ would be a direct sum of a finite number of generalized eigenspaces. On the other hand the lemma says that $W(\lambda)$ has infinitely many irreducible quotients $\operatorname{ev}_{a_{1}}^{*} V\left(\lambda_{1}\right) \otimes \cdots \otimes$ $\operatorname{ev}_{a_{k}}^{*} V\left(\lambda_{k}\right)$ and a simple calculation shows that the eigenvalues of $L(\mathfrak{h})$ on the image of $w_{\lambda}$ in each of these quotients is different.

5.5. Let us look at a further consequence of this lemma. Recall the modules $V(\lambda, \mu, a)$ defined in Section 4.4. If $\lambda-\mu \in Q^{+}$, then with a little computation, one can prove that $V(\lambda, \mu, a)$ is generated as a $L(\mathfrak{g})$-module by the element $v_{\lambda} \in V(\lambda)$ and that $L\left(\mathfrak{n}^{+}\right) v_{\lambda}=0$ and $\operatorname{dim} V(\lambda, \mu, a)_{\lambda}=1$, if $\lambda \neq \mu$. Hence $\operatorname{Hom}_{L(\mathfrak{g})}(W(\lambda), V(\lambda, \mu, a)) \neq 0$ and so $W(\lambda)$ also has cyclic reducible (indecomposable) finite-dimensional quotients. It is reasonable therefore, to ask if there are a family of maximal or universal finite dimensional quotients of $W(\lambda)$ : a family of finite-dimensional quotients $V$ which are cyclic and have $\operatorname{dim} V_{\lambda}=1$ and are such that any other such finite-dimensional quotient of $W(\lambda)$ is a quotient of one of these. The answer is yes and these are called the local Weyl modules and we shall spend some time 
discussing these modules. We remark that it is these local Weyl modules which appear as the $q=1$ limits of representations of quantum affine algebras.

5.6. The module $W(\lambda)$ admits a right $L(\mathfrak{h})$-module structure, given by

$$
\left(y w_{\lambda}\right) u=y u w_{\lambda}, \quad y \in \mathbf{U}(L(\mathfrak{g})), \quad u \in L(\mathfrak{h}),
$$

and hence $W(\lambda)$ is a $(L(\mathfrak{g}), L(\mathfrak{h}))$-bimodule. Given $\chi: L(\mathfrak{h}) \rightarrow \mathbb{C}$, set

$$
W(\lambda, \chi)=W(\lambda) \otimes_{L(\mathfrak{h})} \mathbb{C}_{\chi},
$$

where $\mathbb{C}_{\chi}$ is the one-dimensional $L(\mathfrak{h})$-module defined by $\chi$. The following was proved in 33].

Theorem. Let $\lambda \in P^{+}$and $\chi \in L(\mathfrak{h})^{*}$. Then the module $W(\lambda, \chi)$ is finite-dimensional and is non-zero iff there exists $k \in \mathbb{N}, \lambda_{1}, \cdots, \lambda_{k} \in P^{+}$and $a_{1}, \cdots, a_{k} \in \mathbb{C}^{\times}$such that

$$
\chi\left(h \otimes t^{s}\right)=\sum_{j=1}^{k} \lambda_{j}(h) a_{j}^{s} .
$$

Moreover if $(\lambda, \chi)$ and $\left(\lambda^{\prime}, \chi^{\prime}\right)$ are such that $W(\lambda, \chi)$ and $W\left(\lambda^{\prime}, \chi^{\prime}\right)$ are non-zero, then

$$
W(\lambda, \chi) \cong W\left(\lambda^{\prime}, \chi^{\prime}\right) \Longleftrightarrow \lambda=\lambda^{\prime}, \quad \chi=\chi^{\prime}
$$

The notation used here, although different from that used in [33], is consistent with the notation of the previous sections of these notes.

5.7. The local Weyl modules have a tensor product factorization proved in [33] which is similar to that given in Theorem 4.1. For $\lambda \in P^{+}$and $a \in \mathbb{C}^{\times}$, let $\chi_{\lambda, a} \in L(\mathfrak{h})^{*}$ be given by $\chi_{\lambda, a}\left(h \otimes t^{r}\right)=a^{r} \lambda(h)$.

Proposition. Any non-zero local Weyl module is isomorphic to a tensor product of the form $W\left(\lambda_{1}, \chi_{\lambda_{1}, a_{1}}\right) \otimes \cdots \otimes W\left(\lambda_{k}, \chi_{\lambda_{k}, a_{k}}\right)$ for some $\lambda_{s} \in P^{+}, a_{s} \in \mathbb{C}^{\times}, 1 \leq s \leq k$ with $a_{j} \neq a_{m}$ if $j \neq m$.

As a result of the proposition, to understand the $\mathfrak{g}$-module structure or the dimension of the local Weyl modules it suffices to study the modules $W\left(\lambda, \chi_{\lambda, a}\right)$ for $(\lambda, a) \in \mathbb{C}^{\times}$. These questions are addressed in 33,26$], 42$ for $\mathfrak{g}$ of type $A_{1}, A_{n}$ and $A, D, E$ respectively and in these cases the local Weyl modules can be identified with Demazure modules in positive level representations of affine Lie algebras. This is definitely false for non-simply laced algebras where the local Weyl modules are bigger than the Demazure modules. In general the dimension of the local Weyl modules can be deduced from the work of [2]. As a consequence one knows that the dimension of $W\left(\lambda, \chi_{\lambda, a}\right)$ is independent of the choice of $a \in \mathbb{C}^{\times}$which in turn can be used to prove that $W(\lambda)$ is free as a right module for a suitably defined polynomial ring (the quotient of $\mathbf{U}\left(L(\mathfrak{h})\right.$ ) by the annihilator of $w_{\lambda}$ ). 
5.8. The Weyl modules for $\mathfrak{g} \otimes A$ have been defined and studied in [16] for an arbitrary associative, commutative algebra $A$. The results of that paper show that there are many important differences between the study of Weyl modules for the loop algebra and the more general case. As in the case for irreducible modules, one works with maximal ideals, and the local Weyl modules for a fixed $\lambda$ are parametrized by a maximal ideal of $A$. The dimension of the local Weyl modules even in the simplest cases does depend on the maximal ideal even when $A$ is the polynomial ring in two variables and hence these modules are no longer free right modules. There are thus many interesting problems that arise from passing to the higher dimensional cases.

5.9. Local Weyl modules have also been studied in positive characteristic in [49], [50]. As in the case of simple Lie algebras, the definition needs modification, the most crucial one being that the conditions on $L(\mathfrak{h})$ must be replaced with the conditions on the integral imaginary root vectors defined by Garland. The authors also study the question of base change, i.e., the functor of extension of scalars from the category of finite-dimensional $\mathbf{U}(L(\mathfrak{g}))_{\mathbb{K}}$-modules to that of $\mathbf{U}(L(\mathfrak{g}))_{\mathbb{F}}$-modules. For simple Lie algebras, the functor induces a bijection from the set of isomorphism classes of highest-weight modules for $\mathbf{U}(\mathfrak{g})_{\mathbb{K}}$ to that of isomorphism classes of highest-weight modules for $\mathbf{U}(\mathfrak{g})_{\mathbb{F}}$, where $\mathbb{F}$ is an algebraic extension of $\mathbb{K}$. In particular, the characters of the irreducible modules and the Weyl modules for the hyperalgebras $\mathbf{U}(\mathfrak{g})_{\mathbb{L}}$ do not depend on the choice of the field $\mathbb{L}$, but only on its characteristic. In the case of hyper loop algebras, the story is more complicated since the functor does not send irreducible modules to irreducible modules and, hence, does not preserve the length of a module. For more details on this functor in this case see [51.

5.10. An important family of quotients of the Weyl modules are the Kirillov-Reshetikhin $(\mathrm{KR})$-modules. In the quantum case, these modules are very important, since they admit a crystal basis. Most finite-dimensional representations do not admit crystal bases. These modules first arose from the study of solvable lattice models and a good reference is [45] and again more recent references can be found in [21]. The results of [33] show that one can put $q=1$ and get modules for the loop algebra. The KR-modules, for our purposes, can be viewed as quotients of the local Weyl modules $W\left(m \omega_{i}, \chi_{m \omega_{i}, a}\right)$, where $i \in I, m \in \mathbb{N}$ and $a \in \mathbb{C}^{\times}$. They are obtained by imposing a single additional relation:

$$
\left(x_{\alpha_{i}}^{-} \otimes(t-a) w_{m \omega_{i}}\right)=0 .
$$

Amongst other things, one of the interesting problems is to compute the $\mathfrak{g}$-character of this module and this is done in [27] for the classical Lie algebras and for some nodes of the exceptional Lie algebras. These modules come equipped with a $\mathbb{N}$-grading and, in fact, the graded character is computed in [27] and shown to coincide with the conjectural character formulae in [45]. The relationship between the grading and the parameter $q$ that appears in [45], though, is quite mysterious. An obvious question from a mathematical point of view, is what happens when $m \omega_{i}$ is replaced with an arbitrary weight $\lambda \in P^{+}$. Partial answers to this question can be found in [63]. An alternative way to think of KR-modules is as projective modules in a suitable subcategory and this is being explored in ongoing work with Jacob Greenstein. 


\section{Koszul Algebras, Quivers, And highest Weight CATEgOries}

In this section we discuss an approach, developed jointly with Jacob Greenstein in [19, $[20]$, to the graded representation theory of the algebras $\mathfrak{g} \otimes \mathbb{C}[t]$. We follow the axiomatic approach of Cline, Parshall and Scott which has been successful in both the study of $\mathcal{O}$ and representations in characteristic $p$. The basic idea is to look at subcategories, each of which has finitely many simple objects and enough projectives (or injectives), and to relate it to the module category of a finite-dimensional algebra. One can then also study the quiver with relations attached to such a subcategory. It is usual to assume that the subcategory is a block and also that one has a natural ordering of the simple objects in the block. This is usually given by the Bruhat order on the Weyl group, which in turn is related to extensions between Verma modules.

However, in our case, as we have seen in Section 4.7, the blocks of the finite-dimensional representations are very large and this remains true if we restrict our attention to graded representations of $\mathfrak{g} \otimes \mathbb{C}[t]$. Nevertheless, we are able to identify interesting subcategories, which contain finitely many irreducibles and the corresponding (generalized) Kirillov-Reshetikhin modules discussed in Section 6. We are also able to define an order on the simple modules which comes from an understanding of the extensions between simple modules. The endomorphism algebra of such a subcategory is a finite-dimensional algebra given by a quiver with relations. Under further restrictions the endomorphism algebra has a natural grading and using the results of [3] we prove that the grading is Koszul. In many cases (in fact for an infinite family) it is possible [4] to compute the relations explicitly and we give some concrete examples below. The twisted analogs of these results and in fact further generalizations may be found in 24 .

6.1. The algebra $\mathfrak{g}[t]=\mathfrak{g} \otimes \mathbb{C}[t]$ is naturally graded by $\mathbb{N}$ with the $r^{t h}$-graded piece being $\mathfrak{g} \otimes t^{r}$ for $r \in \mathbb{N}$. The enveloping algebra $\mathbf{U}(\mathfrak{g}[t])$ acquires a well-defined natural grading by $\mathbb{N}$ as well: we say that an element of $\mathbf{U}(\mathfrak{g}[t])$ has grade $s$ if it is a linear combination of elements of the form $\left(x_{1} \otimes t^{s_{1}}\right) \cdots\left(x_{k} \otimes t^{s_{k}}\right)$ where $k \in \mathbb{N}, s_{j} \in \mathbb{N}, x_{j} \in \mathfrak{g}, 1 \leq j \leq k$ and $s=s_{1}+\cdots+s_{k}$. Let $\mathfrak{g}[t]_{+}=\mathfrak{g} \otimes t \mathbb{C}[t]$, which is clearly a Lie ideal of $\mathfrak{g}[t]$. Note that $\mathbf{U}(\mathfrak{g}[t]) \cong \mathbf{U}\left(\mathfrak{g}[t]_{+}\right) \otimes \mathbf{U}(\mathfrak{g})$ and that the graded pieces $\mathbf{U}\left(\mathfrak{g}[t]_{+}\right)[r]$ are finite dimensional $\mathfrak{g}$-modules. Hence for all $\lambda \in P^{+}$, we see that $\mathbf{U}(\mathfrak{g}[t])[s] \otimes \mathbf{U}(\mathfrak{g}) V(\lambda)$ is a finite-dimensional $\mathfrak{g}$-module.

Let $\mathcal{G}$ be the category whose objects are $\mathbb{N}$-graded modules $V$ with finite dimensional graded pieces,

$$
\begin{aligned}
& V=\bigoplus_{r \in \mathbb{N}} V[r], \quad \text { all } \operatorname{dim} V[r]<\infty, \\
& \left(\mathfrak{g} \otimes t^{s}\right) V[r] \subset V[r+s], \quad r, s \in \mathbb{N},
\end{aligned}
$$

and the morphisms are given by

$$
\operatorname{Hom}_{\mathcal{G}}(V, W)=\left\{f \in \operatorname{Hom}_{\mathfrak{g}[t]}(V, W): f(V[r]) \subset W[r], \quad r \in \mathbb{N}\right\} .
$$

For $r \in \mathbb{N}$ let $\tau_{r}$ be the grading shift functor $\mathcal{G} \rightarrow \mathcal{G}$, that is for all $V \in \mathrm{Ob} \mathcal{G}, \tau_{r} V$ is isomorphic to $V$ as a $\mathfrak{g}[t]$-module and $\left(\tau_{r} V\right)[s]=V[r+s]$. 
6.2. Let $\mathrm{ev}_{0}: \mathfrak{g}[t] \rightarrow \mathfrak{g}$, given by $x \otimes t^{r} \rightarrow \delta_{r, 0} x$, be the evaluation at zero. For $\lambda \in P^{+}$we can regard the $\mathfrak{g}[t]-$ module $\mathrm{ev}_{0}^{*} V(\lambda)$ as an object of $\mathcal{G}$ by considering it as being concentrated in grade zero. Set $V(\lambda, r)=\tau_{r} \operatorname{ev}_{0}^{*} V(\lambda)$. The projective cover of $V(\lambda, r)$ in $\mathcal{G}$ is

$$
P(\lambda, r)=\tau_{r} \mathbf{U}(\mathfrak{g}[t]) \otimes_{\mathbf{U}(\mathfrak{g})} V(\lambda), \quad P(\lambda, r)[s]=\mathbf{U}(\mathfrak{g}[t])[s-r] \otimes_{\mathbf{U}(\mathfrak{g})} V(\lambda), \quad s \geq r .
$$

The following is not hard to prove.

Proposition. Any irreducible object in $\mathcal{G}$ is isomorphic to $V(\lambda, r)$ for a unique $(\lambda, r) \in$ $P^{+} \times \mathbb{N}$. Moreover, $P(\lambda, r)$ is an indecomposable projective object of $\mathcal{G}$ and has $V(\lambda, r)$ as its unique irreducible quotient via a map $\tau_{r} \pi_{\lambda}$ which maps $1 \otimes v_{\lambda} \rightarrow v_{\lambda}$.

Since $\mathfrak{g}=[\mathfrak{g}, \mathfrak{g}]$ one deduces that the kernel $K(\lambda, r)$ of the canonical surjection $P(\lambda, r) \rightarrow$ $V(\lambda, r)$ is generated by $\tau_{r} \mathbf{U}(\mathfrak{g}[t])[1] \otimes_{\mathbf{U}(\mathfrak{g})} V(\lambda)$, and if we note that $\mathbf{U}(\mathfrak{g}[t])[1] \cong(\mathfrak{g} \otimes t) \mathbf{U}(\mathfrak{g})$, we get

$$
K(\lambda, r)=\tau_{r}(\mathbf{U}(\mathfrak{g}[t])(\mathfrak{g} \otimes t)) \otimes V(\lambda) .
$$

A little more work proves that

$$
\operatorname{Hom}_{\mathcal{G}}(K(\lambda, r), V(\mu, s)) \cong \begin{cases}0, & s \neq r+1 \\ \operatorname{Hom}_{\mathfrak{g}}(\mathfrak{g} \otimes V(\lambda), V(\mu)), & s=r+1\end{cases}
$$

We note the following

Corollary. For all $(\lambda, r),(\mu, s) \in P^{+} \times \mathbb{N}$ we have

$$
\begin{aligned}
& \operatorname{Ext}_{\mathcal{G}}^{1}(V(\lambda, r), V(\mu, s))=0, \quad s \neq r+1, \\
& \operatorname{Ext}_{\mathcal{G}}^{1}(V(\lambda, r), V(\mu, r+1)) \cong \operatorname{Hom}_{\mathfrak{g}}(\mathfrak{g} \otimes V(\lambda), V(\mu)) .
\end{aligned}
$$

Motivated by the previous corollary, we define a partial order $\preceq$ on the index set $P^{+} \times \mathbb{N}$ of the simple modules in $\mathcal{G}$ by extending the cover relation $(\lambda, r) \prec(\mu, s)$ if and only if $s=r+1$ and $\lambda-\mu \in \Phi \sqcup\{0\}$. Since a pair $(\mu, s)$ can cover only finitely many elements it follows that there exist only finitely many elements less than a given element. A subset $\Gamma$ of the poset $P^{+} \times \mathbb{N}$ is called interval closed if for all $(\mu, s) \prec(\nu, k) \prec(\lambda, r)$ with $(\mu, s),(\lambda, r) \in \Gamma$ we have $(\nu, k) \in \Gamma$.

6.3. Even though an object $V$ of $\mathcal{G}$ could be infinite dimensional, one can still talk about the multiplicity of $V(\lambda, r)$ in $V$ by setting

$$
[V: V(\lambda, r)]=\left[V_{\leq r}: V(\lambda, r)\right], \quad V_{\leq r}=\frac{V}{\bigoplus_{s>r} V[s]}
$$

where the right hand side of the preceding equality makes sense since $V_{\leq r}$ is a finitedimensional object of $\mathcal{G}$. If $[V: V(\lambda, r)]>0$, then we call $V(\lambda, r)$ a composition factor of $V$. It follows from the definition of $P(\lambda, r)$ that

$$
[P(\lambda, r): V(\mu, s)]=\operatorname{dim} \operatorname{Hom}_{\mathfrak{g}}\left(\mathbf{U}\left(\mathfrak{g}[t]_{+}\right)[s-r] \otimes V(\lambda), V(\mu)\right) .
$$

Moreover, one can describe the $\mathfrak{g}$-module structure of $\mathbf{U}\left(\mathfrak{g}[t]_{+}\right)$in terms of symmetric powers of the adjoint representation of $\mathfrak{g}$. 
Given a finite interval closed subset $\Gamma$ of $P^{+} \times \mathbb{N}$, let $\mathcal{G}[\Gamma]$ be the full subcategory of $\mathcal{G}$ consisting of modules $V$ whose composition factors all lie in $\Gamma$, that is, $V \in \mathrm{Ob} \mathcal{G}[\Gamma]$ if and only if

$$
[V: V(\mu, s)]>0 \Longrightarrow(\mu, s) \in \Gamma .
$$

Clearly, $\mathcal{G}[\Gamma]$ is closed under extensions, submodules and quotients. Given $V \in \mathrm{Ob} \mathcal{G}$, let $V_{\Gamma}$ be the maximal $\mathfrak{g}[t]$-submodule of $V$ such that

$$
\left[V_{\Gamma}: V(\mu, s)\right]>0 \Longrightarrow(\mu, s) \notin \Gamma,
$$

and set $V^{\Gamma}=V / V_{\Gamma}$. It is clear from the definition that $V^{\Gamma} \in \mathcal{G}[\Gamma]$ but it is generally not true that $V_{\Gamma} \in \mathcal{G}\left[\Gamma^{c}\right]$, where $\Gamma^{c}$ is the complement of $\Gamma$ in $P^{+} \times \mathbb{N}$. However, as the following proposition shows, it is true for modules $P(\mu, s)$, with $(\mu, s) \in \Gamma$, and it is this fact that makes it possible to compute the ext quiver of the endomorphism algebra of the projective generator of the category $\mathcal{G}[\Gamma]$ for finite, interval closed $\Gamma$.

Proposition. Let $\Gamma$ be an interval closed subset of $\mathcal{G}$ and let $(\lambda, r),(\mu, s) \in \Gamma$. Then,

$$
[P(\lambda, r): V(\mu, s)]=\left[P(\lambda, r)^{\Gamma}: V(\mu, s)\right],
$$

or equivalently

$$
\left[P(\lambda, r)_{\Gamma}: V(\mu, s)\right]=0
$$

and hence $P(\lambda, r)_{\Gamma} \in \mathcal{G}\left[\Gamma^{c}\right]$.

6.4. We continue to assume that $\Gamma$ is finite and interval closed and choose $N \in \mathbb{N}$ so that $(\mu, s) \in \Gamma$ only if $s<N$. Set

$$
P(\Gamma)=\bigoplus_{(\lambda, r) \in \Gamma} P(\lambda, r), \quad \mathfrak{A}(\Gamma)=\operatorname{End}_{\mathcal{G}}(P(\Gamma)) .
$$

We have

$$
\mathfrak{A}(\Gamma) \cong \operatorname{End}_{\mathcal{G}}\left(P(\Gamma)_{\leq N}\right) \cong \operatorname{End}_{\mathcal{G}[\Gamma]}\left(P(\Gamma)^{\Gamma}\right),
$$

where the first isomorphism follows easily from the definitions and the second is an application of Proposition 6.3. One then uses standard arguments to prove that $\mathfrak{A}(\Gamma)$ is a finite-dimensional basic algebra whose left module category is equivalent to $\mathcal{G}[\Gamma]$. Moreover, one can also prove that $\mathfrak{A}(\Gamma)$ is a directed quasi-hereditary algebra and an algorithm to compute the Ext-quiver of this algebra along with the number of relations is given in [19]. As $\Gamma$ varies over interval closed sets, one gets many interesting quivers and the algebras $\mathfrak{A}[\Gamma]$ have varying representation type.

We give one specific example, motivated by the study of Kirillov-Reshetikhin modules, where the algebra is tame. Let $\mathfrak{g}$ be of type $D_{n}$ with $n \geq 6$ and use the standard Bourbaki numbering of the Dynkin diagram. In particular, 4 is not a spin node. Let

$$
\Gamma=\left\{\left(2 \omega_{4}, 0\right),\left(\omega_{2}+\omega_{4}, 1\right),\left(2 \omega_{2}, 2\right),\left(\omega_{4}, 2\right),\left(\omega_{1}+\omega_{3}, 2\right),\left(\omega_{2}, 3\right),(0,4)\right\} .
$$

A simple exercise shows that $\Gamma$ is interval closed. With some further work, the results discussed so far can be used to prove that the category $\mathcal{G}[\Gamma]$ is equivalent to the module 
category of the path algebra of the following quiver

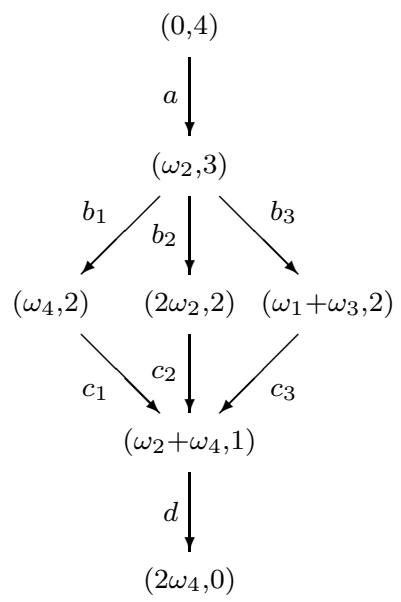

with relations

$$
b_{3} a=0, \quad d c_{3}=0, \quad c_{1} b_{1}+c_{2} b_{2}+c_{3} b_{3}=0 .
$$

In particular, this algebra is quadratic. It can be shown to be tame and of global dimension 2 .

6.5. We now further focus our study on finite-dimensional associative algebras which arise from the full subcategory $\mathcal{G}_{2}$ which consists of objects in $\mathcal{G}$ satisfying

$$
\left(\mathfrak{g} \otimes t^{r}\right) V=0, \quad r \geq 2 .
$$

One could define $\mathcal{G}_{s}$ for $s \in \mathbb{N}$ in the obvious way. For $s=1$ it is easy (and has been said elsewhere in different forms throughout these notes) to see that the category $\mathcal{G}_{1}$ is semi-simple. The irreducible objects are just $\mathrm{ev}_{0}^{*} V(\lambda)$ for $\lambda \in P^{+}$. Thus $\mathcal{G}_{2}$ is the first interesting case which is not semisimple, making it reasonable to limit our attention to this category. It is worth mentioning that restricted Kirillov-Reshetikhin modules in classical types are objects in this category. An object in $\mathcal{G}_{2}$ is actually a representation of the graded truncated Lie algebra $\mathfrak{g} \otimes\left(\mathbb{C}[t] /\left(t^{2}\right)\right)$. This algebra is isomorphic to the semi-direct product $\mathfrak{g} \ltimes \mathfrak{g}_{\text {ad }}$ where $\mathfrak{g}_{\text {ad }}$ is the adjoint representation of $\mathfrak{g}$. Although this algebra is supported only in grades zero and one, the universal enveloping algebra is $\mathbb{N}$-graded and we have an isomorphism of associative algebras

$$
\mathbf{U}\left(\mathfrak{g} \ltimes \mathfrak{g}_{\mathrm{ad}}\right) \cong S\left(\mathfrak{g}_{\mathrm{ad}}\right) \otimes \mathbf{U}(\mathfrak{g}),
$$

and the $r^{\text {th }}$ graded piece is

$$
\mathbf{U}\left(\mathfrak{g} \ltimes \mathfrak{g}_{\mathrm{ad}}\right)[r] \cong S^{r}\left(\mathfrak{g}_{\mathrm{ad}}\right) \otimes \mathbf{U}(\mathfrak{g}) .
$$

Here $S\left(\mathfrak{g}_{\mathrm{ad}}\right)$ is the symmetric algebra of $\mathfrak{g}_{\text {ad }}$ and $S^{r}\left(\mathfrak{g}_{\mathrm{ad}}\right)$ is the $r^{t h}$-symmetric space. One can now use the Koszul complex of $S(\mathfrak{g})$ to construct an explicit projective resolution for any simple object in $\mathcal{G}_{2}$ and to compute all Ext spaces between simple objects in $\mathcal{G}_{2}$.

Proposition. For all $(\lambda, r),(\mu, s) \in P^{+} \times \mathbb{N}$, we have

$$
\operatorname{Ext}_{\mathcal{G}_{2}}^{j}(V(\lambda, r), V(\mu, s)) \cong \begin{cases}0, & j \neq s-r \\ \operatorname{Hom}_{\mathfrak{g}}\left(\bigwedge^{j} \mathfrak{g}_{\mathrm{ad}} \otimes V(\lambda), V(\mu)\right), & j=s-r .\end{cases}
$$


In particular, $\operatorname{Ext}_{\mathcal{G}_{2}}^{j}(M, N)=0$ if $M, N$ are finite dimensional and $j>\operatorname{dim} \mathfrak{g}$.

This proposition strongly suggests that there is an associative algebra with a Koszul grading in the background and we now describe how to find this algebra.

6.6. We first need to refine the partial ordering $\preceq$ on $P^{+} \times \mathbb{N}$. Instead of allowing all possible roots in the cover relation, we restrict ourselves to particular subsets of $\Phi^{+}$obtained as follows. Given $\psi \in P$, let

$$
\Phi_{\psi}^{+}=\left\{\alpha \in R^{+}: \kappa(\psi, \alpha) \geq \kappa(\psi, \beta) \text { for all } \beta \in \Phi\right\}
$$

and define a cover relation by $(\mu, s)$ covers $(\lambda, r)$ iff $s=r+1$ and $\lambda-\mu \in \Phi_{\psi}^{+}$. Let $\leq_{\psi}$ be the corresponding partial order or, equivalently, $(\lambda, r) \leq_{\psi}(\mu, s)$ iff

$$
\mu-\lambda=\sum_{\alpha \in \Phi_{\psi}^{+}} n_{\alpha} \alpha, \quad n_{\alpha} \in \mathbb{N}, \quad \sum_{\alpha \in \Phi_{\psi}^{+}} n_{\alpha}=s-r .
$$

The definition of $\Phi_{\psi}^{+}$ensures that this order is well-defined. The following was proved in [20]:

Theorem. Let $(\lambda, r) \in P^{+} \times \mathbb{N}$ and let $\Gamma=\left\{(\mu, s):(\mu, s) \leq_{\psi}(\lambda, r)\right.$. Then the endomorphism algebra $\mathfrak{A}[\Gamma]$ of the projective generator of $\mathcal{G}_{2}[\Gamma]$ admits a natural Koszul grading and is of global dimension at most the cardinality of $\Phi_{\psi}^{+}$. Moreover the maximal dimension is attained for a suitable choice of $(\lambda, r)$.

One can define certain limits of the algebras as $\Gamma$ varies and construct infinite-dimensional Koszul algebras $\mathfrak{A}_{\psi}$ of left global dimension $\left|\Phi_{\psi}^{+}\right|$. The Koszul dual of these algebras are also studied in 20, although one does not understand the module category of the dual in the context of representations of Lie algebras. We conclude these notes with examples of the quivers associated to the infinite-dimensional Koszul algebras arising from our study. Further details may be found in [44].

6.7. Suppose that $\mathfrak{g}$ is not of type $A$ or $C$ so that there exists a unique $i_{0} \in I$ such that $\theta-\alpha_{i_{0}} \in \Phi^{+}$and then,

$$
\Phi_{\omega_{i_{0}}}^{+}=\left\{\theta, \theta-\alpha_{i_{0}}\right\}
$$

Then every connected subalgebra of $\mathfrak{A}_{\omega_{i}}$ is isomorphic to the path algebra of the translation quiver

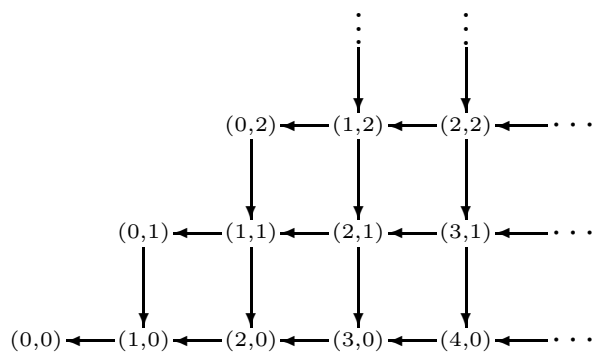

with the mesh relations as indicated. 
6.8. Let $\mathfrak{g}$ be of type $C$. Then $\Phi_{\omega_{2}}^{+}=\left\{\theta, \theta-\alpha_{1}, \theta-2 \alpha_{1}\right\}$ and the quiver of $\mathfrak{A}_{\omega_{2}}$ is the (infinite, if rank of $\mathfrak{g}$ is greater than 2 ) union of connected components of the following two types:
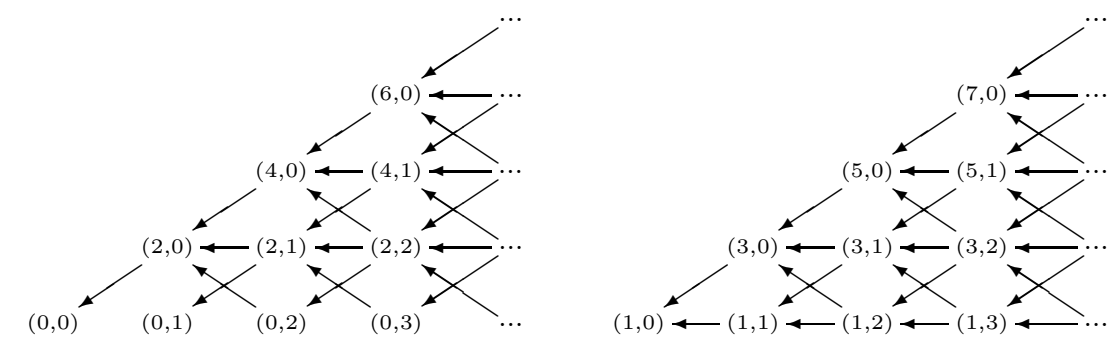

Both are translation quivers with $\tau((m, n))=(m, n-2), m>0, n \geq 2$. The relations are: the commutativity relations in
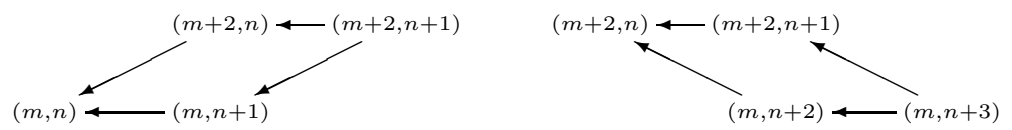

for all $m>0, n \in \mathbb{Z}_{+}$, the zero relations $(2, n) \leftarrow(2, n+1) \leftarrow(0, n+3), n \geq 0$ and

$$
\begin{array}{r}
m^{2}((m, n) \leftarrow(m+2, n) \leftarrow(m, n+2))-(m+2)^{2}((m, n) \leftarrow(m-2, n+2) \leftarrow(m, n+2)) \\
+(m+1)((m, n) \leftarrow(m, n+1) \leftarrow(m, n+2)), \quad m>1,
\end{array}
$$

and, finally, $((1, n) \leftarrow(3, n) \leftarrow(1, n+2))+2((1, n) \leftarrow(1, n+1) \leftarrow(1, n+2))$. Thus, if $\ell=2$ and $\left|\Phi_{\psi}^{+}\right|>1$, the algebra $\mathfrak{A}_{\psi}$ is the direct sum of two non-isomorphic connected Koszul subalgebras of left global dimension 3 .

6.9. Let $\mathfrak{g}$ be of type $G_{2}$ and suppose that $\alpha_{1}$ is the long simple root. Then $\Phi_{\omega_{1}-\omega_{2}}^{+}=$ $\left\{\alpha_{1}, \theta\right\}$ and the algebra $\mathfrak{A}_{\omega_{1}-\omega_{2}}$ is the direct sum of three isomorphic connected subalgebras with quivers

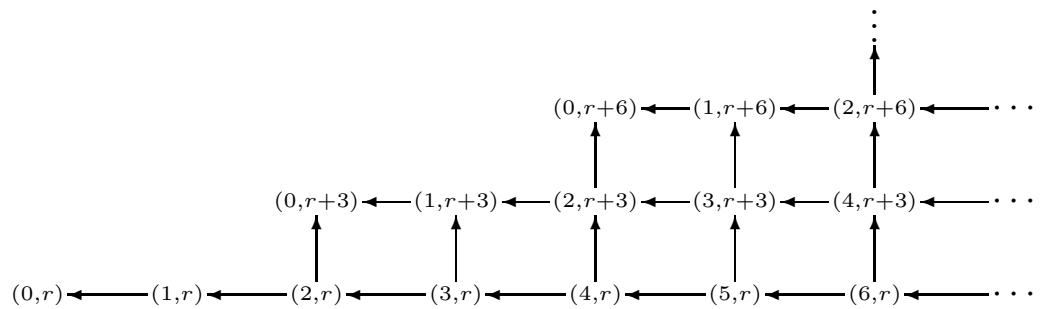

where $0 \leq r \leq 2$. The relations are again the mesh relations, the translation map being

$$
\tau((m, 3 k+r))=(m+3,3(k-1)+r), \quad m \in \mathbb{Z}_{+}, \quad k>0 .
$$

6.10. In conclusion, we note that there is some work [5], [7], [25], [58], [?] on the representation theory of extended affine algebras where the full center does not act trivially. There is also the recent work [41] in which the authors give a representation theoretic way to interpret the third cohomology classes of the double loop algebras. It is perhaps clear by now, that there are many interesting avenues to pursue in the representation theory of affine, 
toroidal and extended affine Lie algebras and while the references are by no means anywhere near exhaustive they should provide the reader with some pointers to the literature.

Vyjayanthi Chari

Department of Mathematics

University of California

Riverside, CA 92521 USA

chari@math.ucr.edu

\section{REFERENCES}

[1] Punita Batra and Tanusree Pal, Representations of Graded Multi-Loop Lie Algebras, arXiv:0706.0448.

[2] J. Beck and H. Nakajima, Crystal bases and two-sided cells of quantum affine algebras, Duke Math. J. 123 (2004), no. 2, 335-402.

[3] A. Beilinson, V. Ginzburg and W. Soergel, Koszul duality patterns in representation theory, J. Amer. Math. Soc. 9 (1996), no. 2, 473527.

[4] A. Beilinson and J. Bernstein, Localisation de $\mathfrak{g}$-modules, C. R.Math.Acad.Sci.Paris 292 (1981), 15-18.

[5] S. Berman, Y. Billig and J. Szmigielski, Vertex operator algebras and the representation theory of toroidal algebras, in Recent developments in infinite-dimensional Lie algebras and conformal field theory (Charlottesville, VA, 2000), pp. 126, Contemp. Math. 297, Amer. Math. Soc., Providence, RI, 2002

[6] J. Bernstein, I. Gelfand and S. Gelfand, A certain category of g-modules, (Russian) Funkcional. Anal. i Prilozen. 10 (1976), no. 2, 18.

[7] Yu. Billig, Representations of toroidal extended affine Lie algebras, J. Algebra 308 (2007) 252269. [Br] L. Breen, Theor

[8] J. L. Brylinski amd M. Kashiwara, Kazhdan-Lusztig conjecture and holonomic systems, Invent. Math 64 (1981), 387-410.

[9] R. E. Borcherds, Vertex algebras, Kac-Moody algebras, and the Monster, Proc. Natl. Acad. Sci. USA 83 (1986), 3068-3071.

[10] N. Bourbaki, Elements de mathematique: Groupes et algèbres de Lie, Hermann, Paris, 1958.

[11] F. Brenti, Kazhdan-Lusztig polynomials: history, problems and combinatorial invariance, Sem. Lothar.Combin., 49 (2002), Art. B49b, 30pp.

[12] R. W. Carter, Lie Algebras of Finite and Affine Type, Cambridge Studies in Advanced Mathematics, 2005.

[13] S. Capparelli, J. Lepowsky and A. Milas, The Rogers-Ramanujan recursion and intertwining operators, Commun. Contemp. Math. 5 (2003), 947-966.

[14] V. Chari, Integrable representations of affine Lie-algebras, Invent. Math. 85 (1986), 317-335.

[15] V. Chari, Ghislain Fourier, Prasad Senesi, Weyl modules for the twisted loop algebras, J. Algebra 319 (2008), no. 12, 50165038.

[16] V. Chari, G. Fourier and T. Khandai A categorical approach to Weyl modules, arXiv:0906.2014

[17] V. Chari and J. Greenstein, An application of free Lie algebras to current algebras and their representation theory Contemporary Mathematics, 392 (2005) 15-31.

[18] V. Chari and J. Greenstein, Graded level zero integrable representations of affine Lie algebras, Trans. of the AMS $\mathbf{3 6 0}$ (2008), no. 6, 2923-2940

[19] V. Chari and J. Greenstein, Current algebras, highest weight categories and quivers, Adv. in Math. 216 (2007), no. 2, 811-840.

[20] V. Chari and J. Greenstein, A family of Koszul algebras arising from finite-dimensional representations of simple Lie algebras, Adv. in Math. 220 (2009), no. 4, 1193-1221.

[21] V.Chari and D. Hernandez, Beyond Kirillov Reshetikhin modules, Contemporary Mathematics, 506, 2010.

[22] V.Chari and S.Ilangovan, On the Harish-Chandra Homomorphism for Infinite-Dimensional Lie Algebras, J. Algebra, 1984, p.476-490

[23] V. Chari and N. Jing, Realization of level one representations of $U_{q}(\hat{g})$ at a root of unity, Duke Math. J. 108 (2001), no. 1, 183-197. 
[24] V.Chari, A.Khare and T.B. Ridenour, in preparation.

[25] V. Chari and T. Le, Representations of double affine Lie algebras, A tribute to C. S. Seshadri (Chennai, 2002), Trends Math., Birkhäuser, Basel, 2003, pp. 199-219.

[26] V. Chari and S. Loktev, Weyl, Demazure and fusion modules for the current algebra of $s l_{r+1}$. Adv. in Math., 207, (2006), Issue 2, 928-960.

[27] V. Chari and A. Moura, Spectral characters of finite-dimensional representations of affine algebras, J. Algebra 279 (2004), no. 2, 820839.

[28] V. Chari and A. Moura, The restricted Kirillov-Reshetikhin modules for the current and twisted current algebras, Commun.Math.Phys. 266 (2006) 431-454.

[29] V. Chari and A. Pressley, New unitary representations of loop groups, Math. Ann. 275 (1986), 87-104.

[30] V. Chari and A. Pressley, Integrable representations of twisted affine Lie algebras, J. Alg. 113 no.2 (1988), 438-464

[31] V. Chari and A. Pressley, Quantum affine algebras, Comm. Math. Phys. 142 (1991), no. 2, 261-283.

[32] V. Chari and A. Pressley, Quantum affine algebras and their representations, Representations of groups (Banff, AB, 1994), CMS Conf. Proc., vol. 16, Amer. Math. Soc., Providence, RI, 1995, pp. 59-78

[33] V. Chari and A. Pressley, Weyl modules for classical and quantum affine algebras, Represent. Theory 5 (2001), 191-223 (electronic).

[34] E. T. Cline, B. J. Parshall and L.L. Scott, Finite-dimensional algebras and highest weight categories, J. Reine. Angew. Math, 391, (1988), 85-99.

[35] V.V.Deodhar, O. Gaber and V.Kac, Structure of some categories of representations of infinitedimensional Lie algebras, Adv. in Math. 45 (1982), 92-116.

[36] V. G. Drinfeld, A new realization of Yangians and of quantum affine algebras, Dokl. Akad. Nauk SSSR 296 (1987), no. 1, 13-17.

[37] P. Etingof and A. Moura, Elliptic central characters and blocks of finite dimensional representations of quantum affine algebras, Represent. Theory 7, (2003), 346373.

[38] I. B. Frenkel and N. Jing, Vertex representations of quantum affine algebras, Proc. Nat. Acad. Sci. U.S.A. 85 (1988), no. 24, 9373-9377.

[39] I. B. Frenkel, V. G. Kac, Basic representations of affine Lie algebras and dual resonance models, Invent. Math. 62 (1980), 23-66.

[40] I. Frenkel, J. Lepowsky, and A. Meurman, Vertex operator algebras and the monster, Academic Press, New York, 1988.

[41] E. Frenkel and X.Zhu, Gerbal Representations of Double Loop Groups arXiv:0810.1487.

[42] G. Fourier and P. Littelmann Weyl modules, Demazure modules, KR-modules, crystals, fusion products and limit constructions Advances in Mathematics 211 (2007) 566593. 567.

[43] H. Garland, The arithmetic theory of loop algebras, J. Algebra, 53, (1978), 480-551.

[44] J. Greenstein, Quivers with relations arising from Koszul algebras of g-invariants, Journal of Algebra 322 (2009), no. 12, 4430-4478.

[45] G. Hatayama, A. Kuniba, M. Okado, T. Takagi and Y. Yamada, Remarks on fermionic formula, in Recent developments in quantum affine algebras and related topics (Raleigh, NC, 1998), 243-291, Contemp. Math., 248, Amer. Math. Soc., Providence, RI (1999).

[46] J.E.Humphreys, Introduction to Lie Algebras and Representation Theory, Springer, 1968

[47] J.E.Humphreys, Representations of semisimple Lie Algebras in the BGG category $\mathcal{O}$, Graduate Studies in Matheamtics, Volume 94, American Mathematical Society, 2008.

[48] J. Jantzen, Representations of algebraic groups, Academic Press (1987).

[49] D. Jakelic and A.A. Moura Finite-Dimensional Representations of Hyper Loop Algebras, Pacific J. Math. 233 (2007), 371-402.

[50] D. Jakelic and A.A. Moura, Finite-dimensional representations of hyper loop algebras over non algebraicaly closed fields, Algebras and Representation Theory published online first DOI 10.1007/s10468008-9122-5.

[51] D. Jakelic and A.A. Moura, On multiplicity problems for finite-dimensional representations of hyper loop algebras, Contemp. Math. 483 (2009), 147-159.

[52] V. G.Kac, Infinite-dimensional Lie algebras, 3rd.ed. Cabridge University Press, 1990. 
[53] V. G. Kac, Vertex algebras for beginners, University Lecture Series, vol. 10, Amer. Math. Soc., Providence, RI, 1996; 2nd ed., 1998.

[54] M. Kashiwara, On level-zero representations of quantized affine algebras, Duke Math. J. 112 (2002), no. $1,117-175$

[55] D.Kazhdan and G.Lusztig, Representations of Coxeter groups and Hecke algebras, Invent.Math 53 (1979), 165-184.

[56] Ryosuke Kodera, Extensions between finite-dimensional simple modules over a generalized current Lie algebra, arXiv:0908.3738.

[57] B. Kostant, Groups over Z, Algebraic Groups and Discontinuous Subgroups, Proc. Symp. Pure Math. IX, Providence, AMS (1966).

[58] T. Larsson, Extended diffeomorphism algebras and trajectories in jet space, Commun. Math. Phys. 214 (2000) 469491.

[59] M. Lau, Representations of multi-loop algebras, math. RT/ arXiv:0811.2011v2.

[60] J. Lepowsky and R. L. Wilson, Construction of the affine Lie algebra $A_{1}^{(1)}$, Commun. Math.Phys. 62 (1978), 43-53.

[61] G. Lusztig, Quantum deformations of certain simple modules over enveloping algebras, Adv. in Math. 70 (1988), no. 2, 237-249.

[62] D. Mitzman, Integral bases for affine Lie algebras and their universal enveloping algebras, Contemp. Math 40 (1985).

[63] Adriano Moura, Restricted limits of minimal affinizations, Pacific J. Math. 244 (2010), No. 2, 359-397

[64] R.V. Moody, S.E. Rao and T. Yokonuma, Toroidal Lie algebras and vertex representations, Geom. Dedicata 35 (1990) 283307.

[65] H. Nakajima, Quiver varieties and finite-dimensional representations of quantum affine algebras, J. Amer. Math. Soc. 14 (2001), no. 1, 145-238 (electronic).

[66] E. Neher, A. Savage and P. Senesi, Irreducible finite-dimensional representations of equivariant map algebras, arXiv:0906.5189.

[67] S.E. Rao, On representations of loop algebras, Comm. Algebra 21 (1993), 2131-2153.

[68] S. E. Rao, Classification of irreducible integrable modules for multiloop algebras with finitedimensional weight spaces, Jour. Alg. 246, (2001), 215225.

[69] P. Senesi, A block decomposition of finite-dimensional representations of twisted loop algebras, To appear in Pacific J. Math., preprint available at arXiv:0807.4116v1

[70] W. Soergel, Kategorie O, perverse Garben und Moduln uber den Koinvarianten zur Weylgruppe, J. Amer. Math. Soc. 3 (1990), no. 2, 421445.

[71] C. Stroppel, Category O: quivers and endomorphism rings of projectives, Represent. Theory 7 (2003), 322-345 (electronic) Department of Mathematics,

Vyjayanthi Chari: Department of Mathematics, University of California at Riverside, CA 92521, $U S A$. 